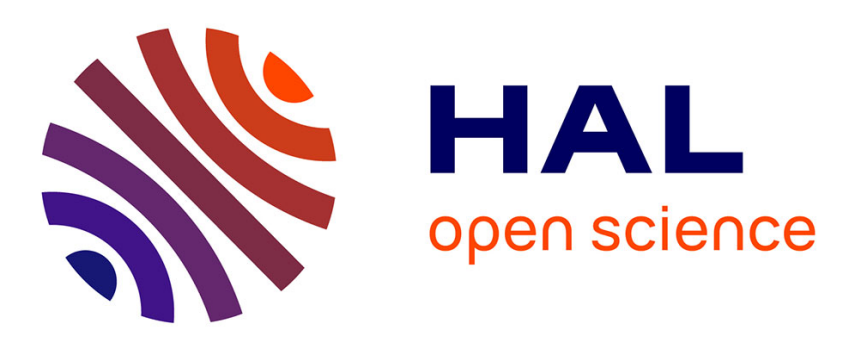

\title{
Augmented Resistive Immersed Surfaces valve model for the simulation of cardiac hemodynamics with isovolumetric phases
}

Alexandre This, Ludovic Boilevin-Kayl, Miguel Angel Fernández, Jean-Frédéric Gerbeau

\section{To cite this version:}

Alexandre This, Ludovic Boilevin-Kayl, Miguel Angel Fernández, Jean-Frédéric Gerbeau. Augmented Resistive Immersed Surfaces valve model for the simulation of cardiac hemodynamics with isovolumetric phases. International Journal for Numerical Methods in Biomedical Engineering, 2020, 36 (3), pp.e3223. 10.1002/cnm.3223 . hal-01944798v2

\section{HAL Id: hal-01944798 \\ https://hal.inria.fr/hal-01944798v2}

Submitted on 10 Dec 2018

HAL is a multi-disciplinary open access archive for the deposit and dissemination of scientific research documents, whether they are published or not. The documents may come from teaching and research institutions in France or abroad, or from public or private research centers.
L'archive ouverte pluridisciplinaire HAL, est destinée au dépôt et à la diffusion de documents scientifiques de niveau recherche, publiés ou non, émanant des établissements d'enseignement et de recherche français ou étrangers, des laboratoires publics ou privés. 
DOI: $\mathrm{xxx} / \mathrm{xxxx}$

\title{
Augmented Resistive Immersed Surfaces valve model for the simulation of cardiac hemodynamics with isovolumetric phases
}

\author{
Alexandre This*1,2,3 | Ludovic Boilevin-Kayl ${ }^{2,3}$ | Miguel A. Fernández ${ }^{2,3}$ | Jean-Frédéric Gerbeau ${ }^{2,3}$
}

\author{
${ }^{1}$ Medisys, Philips Research, 92150 Suresnes, \\ France \\ ${ }^{2}$ Inria Paris, 75012 Paris, France \\ ${ }^{3}$ Sorbonne Université, UMR 7598 LJLL, \\ 75005 Paris, France
}

Correspondence

*Email: alexandre.this@philips.com

\begin{abstract}
In order to reduce the complexity of heart hemodynamics simulations, uncoupling approaches are often considered for the modeling of the immersed valves as an alternative to complex fluid-structure interaction (FSI) models. A possible shortcoming of these simplified approaches is the difficulty to correctly capture the pressure dynamics during the isovolumetric phases. In this work, we propose an enhanced resistive immersed surfaces (RIS) model of cardiac valves which overcomes this issue. The benefits of the model are investigated and tested in blood flow simulations of the left heart.
\end{abstract}

\section{KEYWORDS:}

Heart hemodynamics, Resistive immersed surfaces, One-way uncoupling

\section{1 | INTRODUCTION}

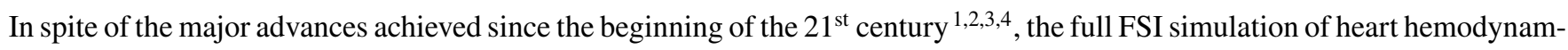
ics remains a complex and challenging problem. Among the main difficulties, we can mention the large structural displacements of the interfaces, the topological changes (when solids come into contact) and the induced high pressure-drops. In order to reduce the complexity of this coupled problem, several alternative models have been proposed in the literature $\frac{516778}{\text {. The fundamental }}$ idea of these simplified approaches consists in combining a reduced modeling of the valves dynamics with a kinematic uncoupling of the cardiac hemodynamics and electromechanics (i.e., displacement fields, coming either from measurements or from simulations, are imposed on the boundaries of the fluid cavities).

These simplifications come however at a price: since the kinematic uncoupling ignores the dynamic balance between cardiac hemodynamics and electromechanics, the fluid pressure within the ventricle is not well defined during the isovolumetric phases (i.e., when all the valves are closed). This explains why this portion of the cardiac cycle is always neglected in the above mentioned uncoupled approaches. Though short-lasting, the isovolumetric phases play a major role in the preservation of a correct unidirectional blood flow within the heart.

In this paper, we propose a new simplified modeling approach which fixes this issue. Starting from the resistive immersed surfaces (RIS) model for valves dynamics ${ }^{5}$, we introduce an additional interface term which controls the ventricle pressure when both valves are closed. This term involves ventricle pressure data coming either from measurements or from external electro-mechanical simulations, hence the terminology augmented RIS model. The fundamental idea of the proposed approach is that, under some basic assumptions and for moderate values of the resistive parameters, the simulated fluid pressure within the ventricle can be regulated in terms of the external pressures, the volume variation and the provided pressure data. Two three-dimensional numerical examples are proposed to test the new model and to assess the validity of its related assumptions. 
The rest of the paper is organized as follows. Section 2 briefly recalls the original RIS model and introduces the new augmented RIS model. In Section 3, two three-dimensional numerical examples are presented. Finally, a summary of the main results and some glimpses of future research are given in Section 4

\section{2 | PROBLEM SETTING AND MATHEMATICAL MODELS}

In this section, we first briefly recall the original RIS model ${ }^{95}$ and its application to a moving cavity enclosed by multiple immersed valves. An estimate of the intracavity pressure in the resulting setting is then provided. This analysis motivates the derivation of the Augmented Resistive Immersed Surfaces (ARIS) model, whose goal is to properly control the intracavity pressure.

\section{1 | Original RIS model}

We consider the flow of a fluid within a moving domain $\Omega(t) \subset \mathbb{R}^{3}$ with $n$ immersed valves. The closed configuration of the valves is given in terms of the surfaces $\Sigma_{i}(t) \subset \Omega, i=1, \ldots, n$. Each surface is assumed to be oriented by a normal $n$. We then define a negative and a positive side via the normal $\boldsymbol{n}^{-} \stackrel{\text { def }}{=} \boldsymbol{n}$ and $\boldsymbol{n}^{+} \stackrel{\text { def }}{=}-\boldsymbol{n}^{-}$. For a given continuous scalar or tensorial field $f$ defined in $\Omega(t)$ (possibly discontinuous across the immersed surface $\Sigma_{i}(t)$ ), we define its positive and negative sided restrictions to $\Sigma_{i}(t)$, denoted respectively by $f^{+}$and $f^{-}$, as $f^{+}(\boldsymbol{x}) \stackrel{\text { def }}{=} \lim _{\xi \rightarrow 0^{+}} f\left(\boldsymbol{x}-\xi \boldsymbol{n}^{+}\right)$and $f^{-}(\boldsymbol{x}) \stackrel{\operatorname{def}}{=} \lim _{\xi \rightarrow 0^{+}} f\left(\boldsymbol{x}-\xi \boldsymbol{n}^{-}\right)$, for all $\boldsymbol{x} \in \Sigma_{i}(t)$.

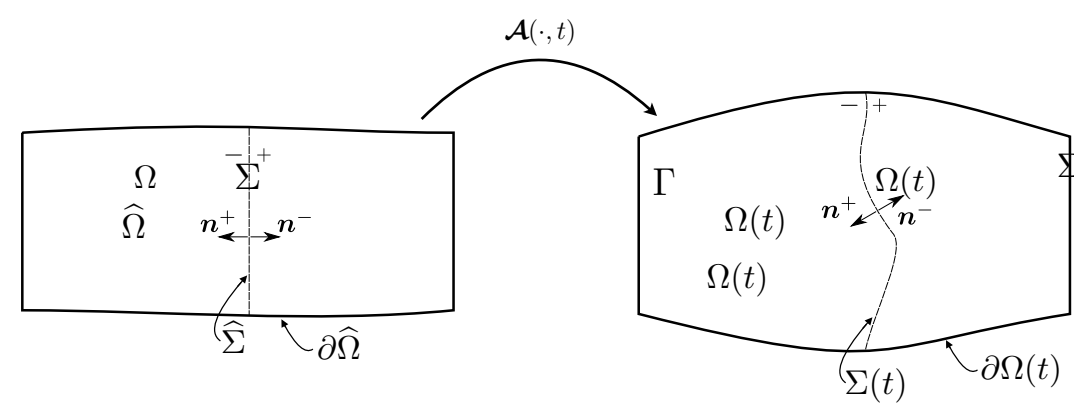

FIGURE 1 Geometric description with a single valve and a moving domain.

The current configuration, in time, is parametrized as $\Omega(t)=\mathcal{A}(\widehat{\Omega}, t)$, where $\widehat{\Omega}$ is the reference configuration and $\mathcal{A}$ : $\widehat{\Omega} \times \mathbb{R}^{+} \rightarrow \mathbb{R}^{3}$ is the Arbitrary Lagrangian-Eulerian (ALE) one-to-one mapping (see Figure 1 ). Similarly, we have the parametrizations $\partial \Omega(t) \stackrel{\text { def }}{=} \mathcal{A}(\partial \widehat{\Omega}, t)$ and $\Sigma(t) \stackrel{\text { def }}{=} \mathcal{A}(\widehat{\Sigma}, t)$. This mapping is given in terms of the fluid domain displacement $\boldsymbol{d}: \hat{\Omega} \times \mathbb{R}^{+} \rightarrow \mathbb{R}^{3}$ with the expression: $\mathcal{A} \stackrel{\text { def }}{=} \boldsymbol{I}_{\hat{\Omega}}+\boldsymbol{d}$. The displacement field $\boldsymbol{d}$ is given in terms of an arbitrary lifting of the exterior boundary displacement $\left.\boldsymbol{d}\right|_{\partial \Omega(t)}$ through the relation $\boldsymbol{d}=\mathcal{L}\left(\left.\boldsymbol{d}\right|_{\partial \Omega(t)}\right)$, where $\mathcal{L}$ is a well chosen lifting operator from $\partial \Omega(t)$ to $\Omega(t)$. The fluid domain velocity $\boldsymbol{w}: \hat{\Omega} \times \mathbb{R}^{+} \rightarrow \mathbb{R}^{3}$ is then simply defined as $\boldsymbol{w} \stackrel{\text { def }}{=} \frac{\partial \boldsymbol{d}}{\partial t}$. We shall also use the notation $\left.\frac{\partial}{\partial t}\right|_{\mathcal{A}}$ for the ALE time derivative.

The fluid is assumed to be homogeneous, incompressible, viscous and Newtonian. It is described by the incompressible Navier-Stokes equations in ALE formulation. The opening and closing dynamics of the valve can be described in a simplified manner by neglecting the mechanics of the leaflets via the RIS model proposed in ${ }^{[5}$. The idea consists in introducing a resistive surface term $R \delta_{\Sigma}(\boldsymbol{u}-\boldsymbol{w})$ in the fluid momentum equation, where $\delta_{\Sigma}$ denotes the Dirac measure on the immersed surface $\Sigma$ and $R=R(t)$ is a time-dependent function which is null when the valve is open and large when the valve is closed. The resulting problem reads as follows: find the velocity $\boldsymbol{u}=\boldsymbol{u}(\boldsymbol{x}, t): \Omega \times \mathbb{R}^{+} \rightarrow \mathbb{R}^{3}$ and the pressure $p=p(x, t): \Omega \times \mathbb{R}^{+} \rightarrow \mathbb{R}$, such that

$$
\left\{\begin{aligned}
\rho\left(\left.\frac{\partial u}{\partial t}\right|_{\mathcal{A}}+(\boldsymbol{u}-\boldsymbol{w}) \cdot \boldsymbol{\nabla u}\right)-\boldsymbol{\nabla} \cdot \boldsymbol{\sigma}(\boldsymbol{u}, p)+\sum_{i=\{1 \ldots n\}} R_{i} \delta_{\Sigma_{i}}(\boldsymbol{u}-\boldsymbol{w})=f & \text { in } \Omega(t), \\
\boldsymbol{\nabla} \cdot \boldsymbol{u}=0 & \text { in } \Omega(t),
\end{aligned}\right.
$$


where the fluid Cauchy stress tensor is given by

$$
\boldsymbol{\sigma}(\boldsymbol{u}, p) \stackrel{\text { def }}{=} 2 \mu \epsilon(\boldsymbol{u})-p \boldsymbol{I}, \quad \boldsymbol{\epsilon}(\boldsymbol{u}) \stackrel{\text { def }}{=} \frac{1}{2}\left(\boldsymbol{\nabla u}+\nabla \boldsymbol{u}^{\mathrm{T}}\right),
$$

and where $\rho$ and $\mu$ denote, respectively, the fluid density and the fluid dynamic viscosity and $\boldsymbol{I}$ the identity tensor. The above system has to be complemented with appropriate initial and boundary conditions. For the sake of notation, in the rest of the paper, $\Omega$ will refer to $\Omega(t), \partial \Omega$ to $\partial \Omega(t)$ and $\Sigma$ to $\Sigma(t)$.

As pointed out in the original model ${ }^{5}$, if one defines the stress jump:

$$
\llbracket \sigma(u, p) n \rrbracket \stackrel{\text { def }}{=} \sigma\left(u^{-}, p^{-}\right) n^{-}+\sigma\left(u^{+}, p^{+}\right) n^{+},
$$

the system (1) enforces the interface conditions

$$
\llbracket \sigma(u, p) n \rrbracket=-R_{i}(u-w) \quad \text { on } \quad \Sigma_{i},
$$

for $i=\{1, \ldots, n\}$. Two asymptotic behaviors are then deduced: when $R_{i}$ is large, $\boldsymbol{u}=\boldsymbol{w}$ is enforced on the moving surface $\Sigma_{i}$, whereas when $R_{i}$ is close to 0 , the flow does not 'see' $\Sigma_{i}$ anymore and there is no pressure drop across it. When $R_{i}$ is between these two extremes, $\Sigma_{i}$ acts as a porous surface $\frac{910}{12}$ via the interface Robin condition (2).

\subsection{Estimate of the pressure within an enclosed cavity}

Based on the original RIS model (1), let $\Omega$ denote a moving domain, composed of three fluid sub-domains $\Omega \stackrel{\text { def }}{=} \Omega_{1} \cup \Omega_{2} \cup \Omega_{3}$, as depicted in Figure 2 We assume that $\Omega_{1}$ shares an interface with $\Omega_{2}$, denoted as $\Sigma_{12}$, and that $\Omega_{2}$ also shares another interface with $\Omega_{3}$, denoted as $\Sigma_{23}$. The domains $\Omega_{1}$ and $\Omega_{3}$ do not share any interface. The opening and closing dynamics of $\Sigma_{12}$ and $\Sigma_{23}$ are described using the RIS model.

The exterior boundary of $\Omega$ is divided into non-overlapping subsets $\partial \Omega_{, \mathrm{fD}}$ and $\partial \Omega_{{ }_{, \mathrm{N}}}$ as depicted in Figure 2 No-slip Dirichlet boundary conditions are imposed on $\partial \Omega_{\cdot, \mathrm{D}}=\partial \Omega_{1, \mathrm{D}} \cup \partial \Omega_{2, \mathrm{D}} \cup \partial \Omega_{3, \mathrm{D}}$ and Neumann normal boundary conditions are imposed on $\partial \Omega_{\cdot, \mathrm{N}}=\partial \Omega_{1, \mathrm{~N}} \cup \partial \Omega_{3, \mathrm{~N}}$. The body force $\boldsymbol{f}$ is assumed to be equal to $\mathbf{0}$.

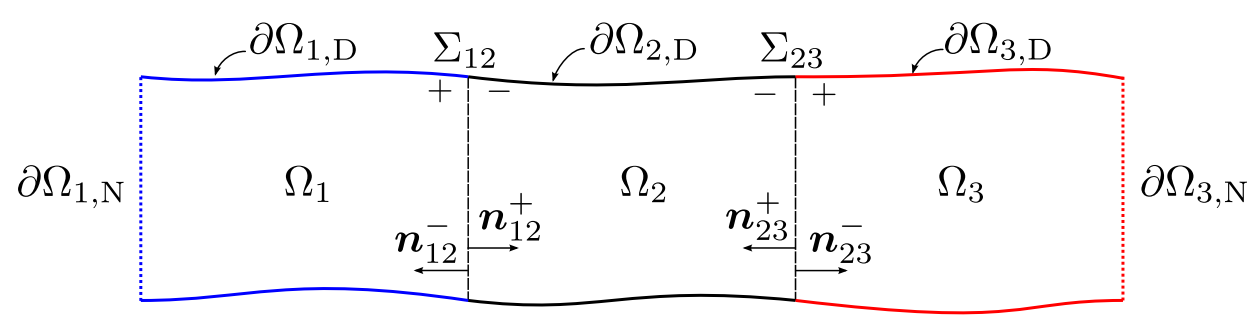

FIGURE 2 Geometrical domain $\Omega$ considered for the problems (3) and (9).

The following problem is therefore considered: find the velocity $\boldsymbol{u}=\boldsymbol{u}(\boldsymbol{x}, t): \Omega \times \mathbb{R}^{+} \rightarrow \mathbb{R}^{3}$ and the pressure $p=p(\boldsymbol{x}, t)$ : $\Omega \times \mathbb{R}^{+} \rightarrow \mathbb{R}$, such that

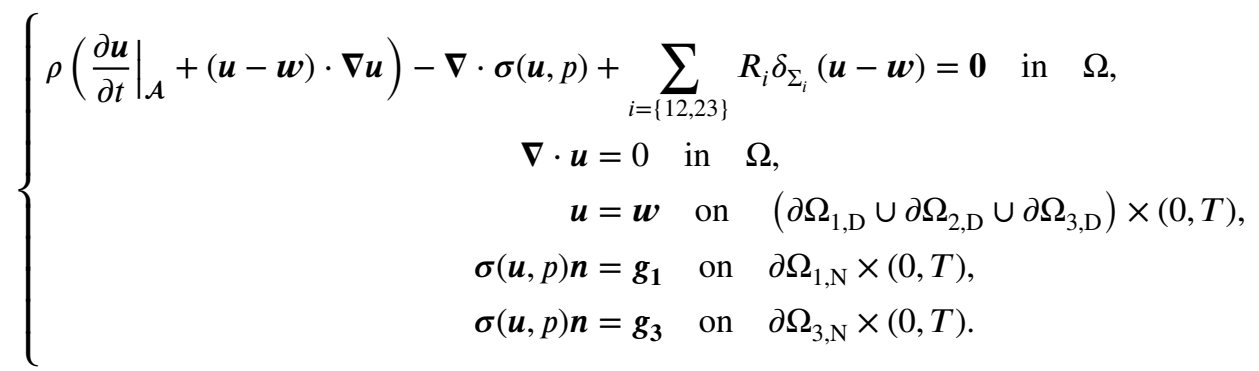

The above system has to be complemented with an appropriate initial condition. The main difficulty which arises in the description of the isovolumetric phases using a uncoupling approach is that the ventricular pressure is defined up to a constant, since the fluid velocity is prescribed on the boundary of the enclosed cavity. This issue is circumvented in the original RIS model 
(1) thanks to the interface Robin condition (2), for moderate values of $R_{i}$. However, as we shall discuss below, this results in an arbitrarily fixed internal pressure.

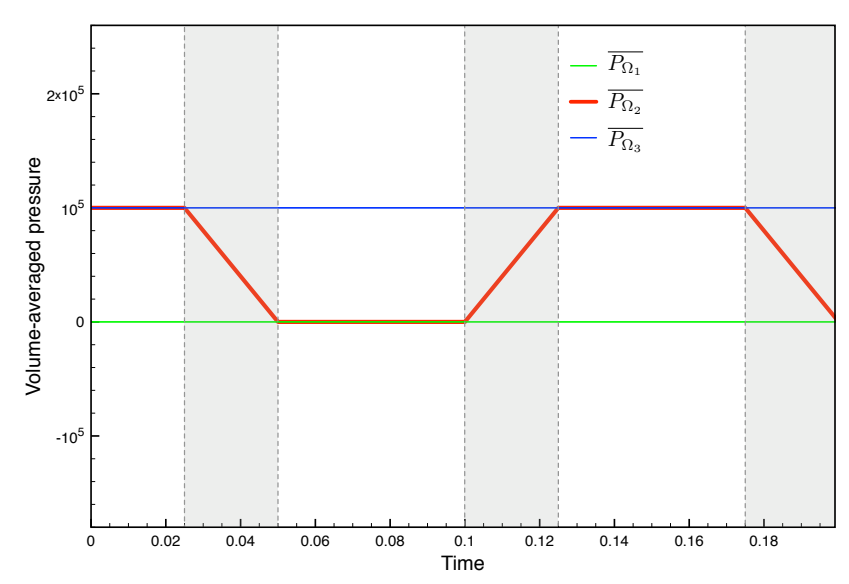

(a) Expected results.

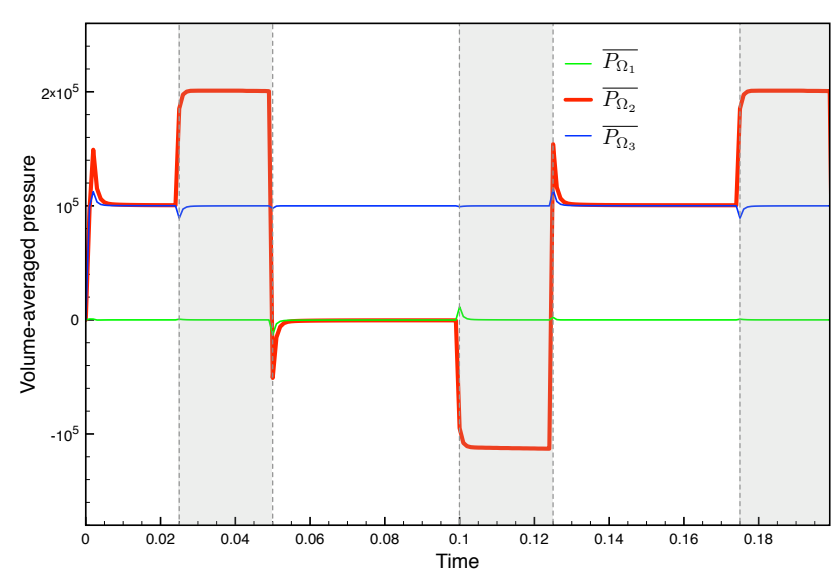

(b) Typical obtained results.

FIGURE 3 Time history of the pressures. The grey areas delimit the isovolumetric phases.

For instance, Figures $3 \mathrm{a} 3 \mathrm{~b}$ depict, respectively, the expected pressures and the typical computed ones, in a simple toy problem based on the domain depicted in Figure 2 and described later in Section 3.1 Results portrayed on Figure 3b have been obtained by using the setting (3). Outside the chosen isovolumetric phases delimited by the gray areas, the computed pressures match the reference ones depicted on Figure $3 \mathrm{a}$ On the other hand, when the middle cavity $\Omega_{2}$ is closed, its inner computed volumeaveraged pressure $\overline{P_{\Omega_{2}}}$ is clearly different from the expected one. It can be noted that, during these isovolumetric phases, $\overline{P_{\Omega_{2}}}$ reaches an arbitrary value, outside of the interval $\left[\overline{P_{\Omega_{1}}}, \overline{P_{\Omega_{3}}}\right]$.

In the following analysis, we provide an estimate of the resulting pressure inside $\Omega_{2}$ in the situation where both $R_{12}$ and $R_{23}$ belong to $] 0,+\infty[$. We consider two simplifying asumptions:

(H1) the viscous contribution to the normal stress is considered negligible on the valves;

(H2) the pressure inside the cavities are considered approximately constant in space when the valves are closed, namely,

$$
p(x, t) \simeq P_{\Omega_{i}}(t) \quad \forall x \in \Omega_{i}, \forall i \in\{1,2,3\} .
$$

Proposition 1. Under assumptions (H1) and (H2), the pressure inside the cavity $\Omega_{2}$ can be estimated as

$$
P_{\Omega_{2}} \simeq \frac{\varepsilon_{12}\left|\Sigma_{12}\right|}{K} P_{\Omega_{1}}+\frac{\varepsilon_{23}\left|\Sigma_{23}\right|}{K} P_{\Omega_{3}}-\frac{1}{K} \int_{\partial \Omega_{2}} \boldsymbol{w} \cdot \boldsymbol{n},
$$

where $\varepsilon_{i} \stackrel{\text { def }}{=} \frac{1}{R_{i}}, K \stackrel{\text { def }}{=}\left(\varepsilon_{12}\left|\Sigma_{12}\right|+\varepsilon_{23}\left|\Sigma_{23}\right|\right)$ and $\left|\Sigma_{i}\right|$ stands for the measure of the surface $\Sigma_{i}$.

Proof. We consider the interface condition on $\Sigma_{i}$ :

$$
\llbracket \sigma(u, p) n \rrbracket=-R_{i}(u-w) \quad \text { on } \quad \Sigma_{i}
$$

Using assumption (H1) and rearranging the terms, we obtain

$$
\boldsymbol{u} \simeq \boldsymbol{w}+\frac{1}{\boldsymbol{R}_{i}}\left(p^{-} \boldsymbol{n}^{-}+p^{+} \boldsymbol{n}^{+}\right) \quad \text { on } \quad \Sigma_{i} .
$$

Multiplying by $\boldsymbol{n}^{-}$and integrating over $\Sigma_{i}$, we get

$$
\int_{\Sigma_{i}} \boldsymbol{u} \cdot \boldsymbol{n}^{-} \simeq \int_{\Sigma_{i}} \boldsymbol{w} \cdot \boldsymbol{n}^{-}+\frac{1}{R_{i}} \int_{\Sigma_{i}}\left(p^{-}-p^{+}\right) .
$$


Using assumption (H2), we finally obtain

$$
\int_{\Sigma_{i}} \boldsymbol{u} \cdot \boldsymbol{n}^{-} \simeq \int_{\Sigma_{i}} \boldsymbol{w} \cdot \boldsymbol{n}^{-}+\frac{1}{R_{i}}\left(p^{-}-p^{+}\right)\left|\Sigma_{i}\right| .
$$

In particular, for $\Sigma_{12}$ and $\Sigma_{23}$, we get

$$
\begin{aligned}
& \int_{\Sigma_{12}} \boldsymbol{u} \cdot \boldsymbol{n}^{-} \simeq \int_{\Sigma_{12}} \boldsymbol{w} \cdot \boldsymbol{n}^{-}+\frac{1}{R_{12}}\left(P_{\Omega_{2}}-P_{\Omega_{1}}\right)\left|\Sigma_{12}\right| \\
& \int_{\Sigma_{23}} \boldsymbol{u} \cdot \boldsymbol{n}^{-} \simeq \int_{\Sigma_{23}} \boldsymbol{w} \cdot \boldsymbol{n}^{-}+\frac{1}{R_{23}}\left(P_{\Omega_{2}}-P_{\Omega_{3}}\right)\left|\Sigma_{23}\right| .
\end{aligned}
$$

Applying the divergence theorem on $(3)_{2}$, we have

$$
\int_{\Omega_{2}} \boldsymbol{\nabla} \cdot \boldsymbol{u}=0 \Leftrightarrow \int_{\partial \Omega_{2, \mathrm{D}}} \boldsymbol{u} \cdot \boldsymbol{n}^{-}+\int_{\Sigma_{12}} \boldsymbol{u} \cdot \boldsymbol{n}^{-}+\int_{\Sigma_{23}} \boldsymbol{u} \cdot \boldsymbol{n}^{-}=0 .
$$

Substituting by $[5],(6)$ and $(3)_{3}$ into (7):

$$
\int_{\partial \Omega_{2}} \boldsymbol{w} \cdot \boldsymbol{n}^{-}+\frac{1}{R_{12}}\left(P_{\Omega_{2}}-P_{\Omega_{1}}\right)\left|\Sigma_{12}\right|+\frac{1}{R_{23}}\left(P_{\Omega_{2}}-P_{\Omega_{3}}\right)\left|\Sigma_{23}\right| \simeq 0 .
$$

Rearranging the equation, we get the the estimation (4). This completes the proof.

It is worth noting that, when the cavity is closed and if the assumptions hold, the pressure $P_{\Omega_{2}}$ is regulated both by the exterior pressures (i.e., with $P_{\Omega_{1}}$ and $P_{\Omega_{3}}$ ) and by the change of the volume of $\Omega_{2}$. In the case of a perfectly incompressible cavity (i.e., when $\int_{\partial \Omega_{2}} \boldsymbol{w} \cdot \boldsymbol{n}=0$ ), a balance of the exterior pressures is reached and is regulated both by the surface area of the immersed surfaces $-\left|\Sigma_{12}\right|$ and $\left|\Sigma_{23}\right|$ - and by the value of their corresponding resistive parameters $R_{i}$.

If the volume of the closed cavity is undergoing change (i.e., $\int_{\partial \Omega_{2}} \boldsymbol{w} \cdot \boldsymbol{n} \neq 0$ ), its pressure will also evolve. Noting that $\boldsymbol{n}$ is the outgoing normal, the dilatation of the cavity (i.e., $\left.\int_{\partial \Omega_{2}} \boldsymbol{w} \cdot \boldsymbol{n}>0\right)$ would induce a negative pressure contribution. On the opposite, a contracting cavity (i.e., $\int_{\partial \Omega_{2}} \boldsymbol{w} \cdot \boldsymbol{n}<0$ ) would result in a positive pressure contribution. Moreover, this pressure change is proportional to $R_{i}$ and inversely proportional to the surface area of the immersed surfaces $-\left|\Sigma_{12}\right|$ and $\left|\Sigma_{23}\right|$. This behavior is coherent with the fact that the original RIS formulation has been designed to model porous media. In the case of an increasing porous surface area, more fluid would pass through, resulting in a lower pressure jump across the surface. On the other hand, in the case of a decreasing porosity, less fluid would pass through, resulting in a higher pressure jump.

While the intracavity pressure has been estimated in a situation where both $R_{12}$ and $R_{23}$ are different from 0 , it has to be noted that this estimation becomes singular when both $R_{12}$ and $R_{23}$ converge to $+\infty$. In this asymptotic case, the fact that the velocity is prescribed on the whole cavity boundary leaves the pressure undetermined and might not be compatible with the incompressibility constraint.

\section{3 | Augmented Resistive Immersed Surface (ARIS) model}

As shown in Section 2.2 an estimation of the pressure $P_{\Omega_{2}}$ has been proposed. This expression involves the distal pressures, the wall velocity, the surface area of the immersed surfaces as well as the value of their resistive parameters. In this section, given a reference time dependent pressure $P^{\star}=P^{\star}(t)$, the objective is to modify the RIS model in order to enforce $P_{\Omega_{2}} \simeq P^{\star}$.

The proposed approach consists in considering an additional normal stress term on each of the immersed surfaces so that the corresponding interface condition becomes:

$$
\llbracket \sigma(u, p) n \rrbracket_{\Sigma_{i}}=-R_{i}(u-w)+g_{\Sigma_{i}} n .
$$


The proposed approach will be referred to as the augmented resistive immersed surface (ARIS) model. Assuming the same setting as the one in Section 2.2 and applying this model on the immersed surfaces $\Sigma_{12}$ and $\Sigma_{23}$, we consider the following problem: find the velocity $\boldsymbol{u}=\boldsymbol{u}(\boldsymbol{x}, t): \Omega \times \mathbb{R}^{+} \rightarrow \mathbb{R}^{3}$ and the pressure $p=p(\boldsymbol{x}, t): \Omega \times \mathbb{R}^{+} \rightarrow \mathbb{R}$, such that

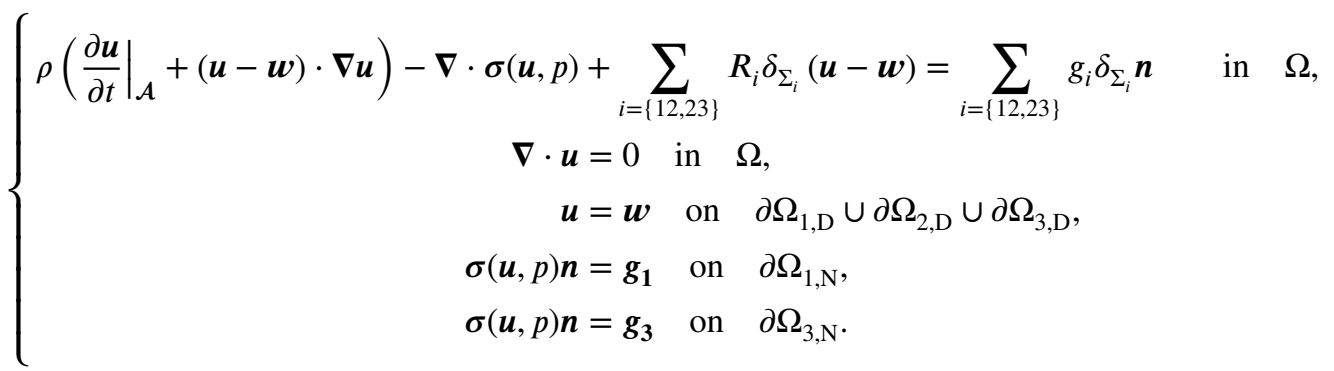

The above system has to be complemented with an appropriate initial condition. The following interface conditions are then satisfied on $\Sigma_{i}$ :

$$
\llbracket u \rrbracket_{\Sigma_{i}}=\mathbf{0}, \quad \llbracket \sigma(u, p) n \rrbracket_{\Sigma_{i}}=-R_{i}(u-w)+g_{\Sigma_{i}} n .
$$

Proposition 2. Let $P^{\star}=P^{\star}(t)$ be a given time-depend function. Using the ARIS model with the stress terms $g_{\Sigma_{12}}$ and $g_{\Sigma_{23}}$ defined as

$$
g_{12}=P_{\Omega_{1}}-P^{\star}-\frac{1}{K} \int_{\partial \Omega_{2}} \boldsymbol{w} \cdot \boldsymbol{n}, \quad g_{23}=P_{\Omega_{3}}-P^{\star}-\frac{1}{K} \int_{\partial \Omega_{2}} \boldsymbol{w} \cdot \boldsymbol{n},
$$

we have the following estimation of the pressure inside the cavity $\Omega_{2}$ :

$$
P_{\Omega_{2}} \simeq P^{\star} .
$$

Proof. By reproducing the proof of Section 2.2 using the interface condition resulting of the proposed ARIS model as a starting point, the pressure estimate $P_{\Omega_{2}}$ obtained is

$$
P_{\Omega_{2}} \simeq \frac{\varepsilon_{12}\left|\Sigma_{12}\right|}{K}\left(P_{\Omega_{1}}-g_{\Sigma_{12}}\right)+\frac{\varepsilon_{23}\left|\Sigma_{23}\right|}{K}\left(P_{\Omega_{3}}-g_{\Sigma_{23}}\right)-\frac{1}{K} \int_{\partial \Omega_{2}} \boldsymbol{w} \cdot \boldsymbol{n} .
$$

Substituting (10) into (12), one readily obtains $(12)$. This completes the proof.

Remark 1. Looking at the pressure estimate [12], it is clear that one can find an infinite amount of pairs $\left\{g_{\Sigma_{12}}, g_{\Sigma_{23}}\right\}$ for which $P_{\Omega_{2}} \simeq P^{\star}$. The proposed pair (10) is nevertheless intuitive as each corrective term counteracts the contribution of the exterior pressure related to the immersed surface on which this term is applied. Moreover, the term related to the cavity volume change is counteracted by both $g_{\Sigma_{12}}$ and $g_{\Sigma_{23}}$ at the same time.

\section{3 | NUMERICAL EXPERIMENTS}

In this section, we illustrate and discuss the benefits brought by the pressure correction described in Section 2.3 in two numerical examples. Problem (9) is discretized in space using $\mathbb{P}_{1} / \mathbb{P}_{1}$ stabilized finite elements for the velocity and the pressure unknowns. The velocity approximations are globally continuous in space while the discrete pressure is allowed to be discontinuous across the immersed surface $\Sigma_{12}$ and $\Sigma_{23}$. This allows to correctly capture the pressure jumps when the valves are closed $\frac{5}{5}$.

As the core motivation of the present work is the simulation of the isovolumetric phases in the ventricle of the left heart, two three-dimensional examples mimicking the behavior of such system are considered. To assess the efficiency of the ARIS model, prescribed reference pressure will be compared with the simulated one in different formulations of the pressure corrective term (10). Finally, the choice of the resistive parameter $R_{i}$ is crucial and requires a specific care. The determination of its value will be discussed, for each numerical example, in the next paragraphs.

\section{1 | Toy problem}

A simple academic problem is designed to reproduce the setting and the problematics described in Section 2 The simplified geometry of the fluid domain is given by $\Omega$, which has the shape of a cylinder, as depicted in Figure $4 \mathrm{a}$ The domain is divided 
into 3 parts, successively denoted as $\Omega_{1}, \Omega_{2}$ and $\Omega_{3}$, as depicted in Figure $4 \mathrm{~b}$. Their dimensions are respectively given by $\left\{r_{\Omega_{1}}=1, l_{\Omega_{1}}=2\right\},\left\{r_{\Omega_{2}}=1, l_{\Omega_{2}}=6\right\}$ and $\left\{r_{\Omega_{3}}=1, l_{\Omega_{3}}=2\right\}$, where $r$ and $l$ stand for, respectively, the radius and the length of the cylindric subdomain. All the units are given in the CGS units system. Two identical planes surfaces are immersed inside the domain: $\Sigma_{12}$ is the interface between $\Omega_{1}$ and $\Omega_{2}$ whereas $\Sigma_{23}$ is the interface between $\Omega_{2}$ and $\Omega_{3}$, as shown in Figure $4 b$

The physical parameters for the fluid are $\rho=1.06$ and $\mu=0.04$. The simulations are carried out for a total time of $t=0.2$ with a time step of $\tau=10^{-3}$. The fluid is initially at rest. The whole computational domain $\Omega$ is made of 7375 tetrahedron. Both immersed surface, $\Sigma_{12}$ and $\Sigma_{23}$, are made of 95 triangular elements.

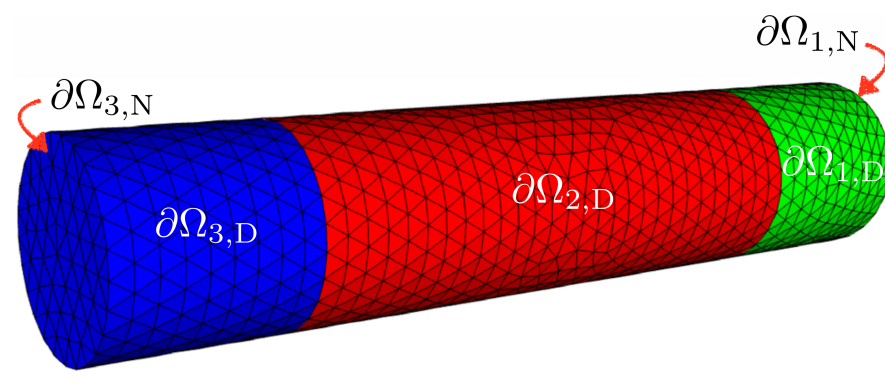

(a) Exterior of the fluid domain.

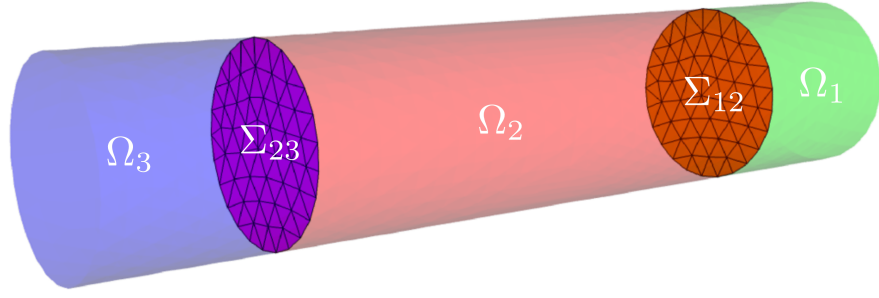

(b) Interior of the fluid domain.

FIGURE 4 Computational domains used for the toy problem model.

A no-slip boundary condition is enforced on the solid wall of the large cylinder $\partial \Omega_{1, \mathrm{D}} \cup \partial \Omega_{2, \mathrm{D}} \cup \partial \Omega_{3, \mathrm{D}}$. The following Neumann conditions are prescribed on the inlet and outlet boundaries:

$$
\begin{array}{lll}
\boldsymbol{\sigma}(\boldsymbol{u}, p) \boldsymbol{n}=\mathbf{0} & \text { on } & \partial \Omega_{1}, \\
\boldsymbol{\sigma}(\boldsymbol{u}, p) \boldsymbol{n}=-10^{5} \boldsymbol{n} & \text { on } & \partial \Omega_{3} .
\end{array}
$$

Moreover, a reference pressure $P^{\star}$, which should be obtained for $P_{\Omega_{2}}$, is used in the pressure corrective term (10). All these pressures have been analytically generated to imitate the main simplified characteristics of a human left heart ${ }^{11}$ and are depicted on Figure 5

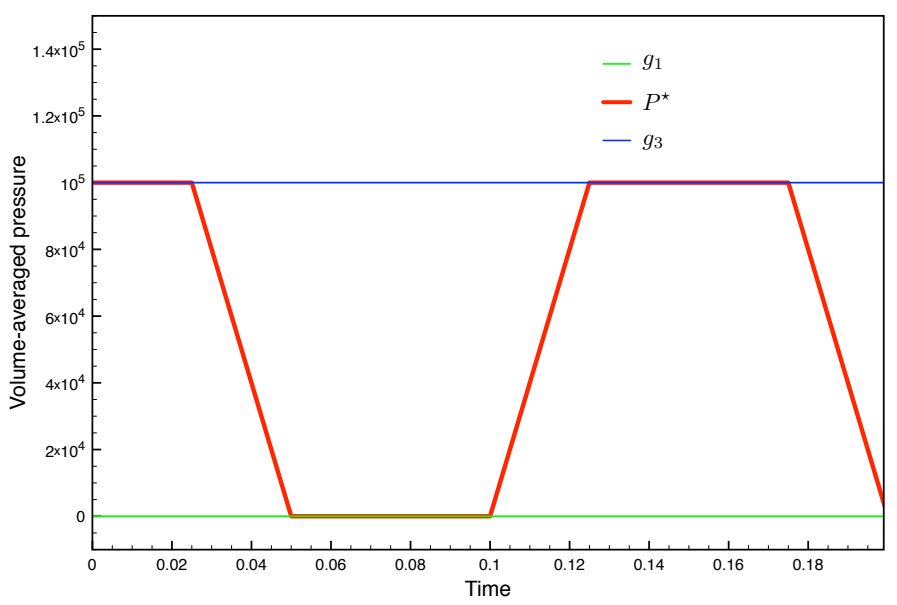

FIGURE 5 Time history of the reference pressures used for each cavity of the toy problem model.

The surface of the middle cavity $\partial \Omega_{2, \mathrm{D}}$ is displaced to mimic the typical behavior of a left ventricle during a heartbeat. In order to address different situations where the incompressibility constraint is broken or not, three different surface velocities, denoted $\boldsymbol{w}_{0}, \boldsymbol{w}_{\text {iso }}$ and $\boldsymbol{w}_{\text {not-iso }}$, evolving with time, are considered. The corresponding displacement field is then obtained thanks 
to a simple backward Euler method. The motion of the full domain is computed by extending the imposed surface displacements within the domain $\Omega$ using an appropriate non-linear lifting operator ${ }^{12}$ (denoted $\mathcal{L}$ in Section 2.1). The surface velocities are given by the following expressions for all $x \in \partial \Omega_{2, \mathrm{D}}$ :

$$
\begin{aligned}
& \boldsymbol{w}_{0}(\boldsymbol{x}, t)=\mathbf{0} \quad \forall t \in \mathbb{R}^{+} . \\
& \boldsymbol{w}_{\text {iso }}(\boldsymbol{x}, t)= \begin{cases}\boldsymbol{0} & \forall t \in \mathbb{R}^{+} \mid\left(R_{12}(t) \neq 0 \text { and } R_{23}(t) \neq 0\right), \\
\boldsymbol{r}(\boldsymbol{x}, t) & \forall t \in \mathbb{R}^{+} \mid\left(R_{12}(t)=0 \text { or } R_{23}(t)=0\right) .\end{cases} \\
& \boldsymbol{w}_{\text {not-iso }}(\boldsymbol{x}, t)= \begin{cases}\boldsymbol{r}(\boldsymbol{x}, t) / 10 & \forall t \in \mathbb{R}^{+} \mid\left(R_{12}(t) \neq 0 \text { and } R_{23}(t) \neq 0\right), \\
\boldsymbol{r}(\boldsymbol{x}, t) & \forall t \in \mathbb{R}^{+} \mid\left(R_{12}(t)=0 \text { or } R_{23}(t)=0\right) .\end{cases}
\end{aligned}
$$

where $\boldsymbol{r}$ is a periodic function of period $T=0.15$, mimicking contraction and dilatation of a pumping heart. This function $\boldsymbol{r}$ is tailored to produce a radial displacement. To obtain a negligible shift of the vertices at the immersed surface level, a gaussian profile is prescribed to get a greater displacement at the center of $\Omega_{2}$ compared with its extremities.

Figures 6a-6c present some typical snapshots of the displacement field obtained with $\boldsymbol{w}_{\text {iso }}$ and applied to the computational domain, respectively, at $t=0$ (initial state), $t=0.04$ (maximum contraction state) and $t=0.115$ (maximum dilatation state).

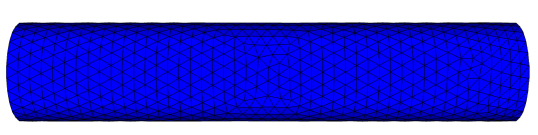

(a) $t=0$.

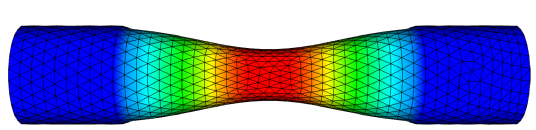

(b) $t=0.04$.

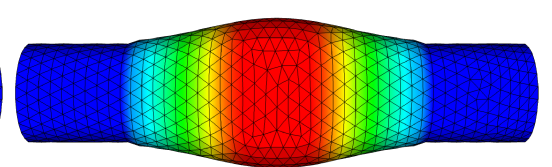

(c) $t=0.115$.

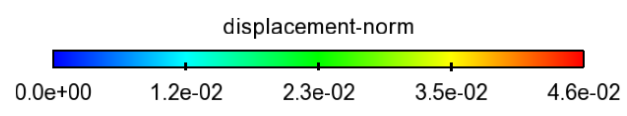

FIGURE 6 Snapshots of the displacement magnitude of the computational domain obtained with $\boldsymbol{w}_{\text {iso }}$ for the toy problem model. The amplitude of the deformation field has been amplified by 10 to increase its visibility.

In this toy problem model, the values of $R_{12}$ and $R_{23}$ are arbitrarily prescribed. Figure 7 a depicts the evolution of the status of the valves and Figure $7 \mathrm{~b}$ relates it to the evolution of the volume of the cavity $\Omega_{2}$. Long contraction and dilatation phases are interspersed with three short isovolumetric phases (respectively defined by the time intervals $[t=0.025, t=0.05]$, $[t=0.1, t=0.125]$ and $[t=0.175, t=0.2])$. During the contraction and dilatation phases, at least one of the two valves is open, while both valves are closed during isovolumetric phases.

The representation of the volume curves corresponding to $\boldsymbol{w}_{\text {iso }}$ and $\boldsymbol{w}_{\text {not-iso }}$ is given on Figure $7 \mathrm{~b} \boldsymbol{w}_{0}$ represents the simple case where the computational domain is not moving. On the other hand, $\boldsymbol{w}_{\text {iso }}$ represents a case with perfect isovolumetric phases while $\boldsymbol{w}_{\text {not-iso }}$ reflects non-perfect isovolumetric phases where a slight residual is introduced when both valves are closed - between $t=0.025$ and $t=0.05$ and between $t=0.1$ and $t=0.125$ - hence inducing a violation of the incompressibility constraint.

The pressure correction is tested using successively the following two terms:

- $g_{\Sigma_{i}, \mathrm{pc}} \stackrel{\text { def }}{=} p^{+}-P^{\star}$ on $\Sigma_{i}$ : the partial corrective term which only accounts for the contribution of the distal pressures.

- $g_{\Sigma_{i}, \mathrm{c}} \stackrel{\text { def }}{=} g_{\Sigma_{i}, \mathrm{pc}}-\frac{1}{K} \int_{\partial \Omega_{2}} \boldsymbol{w} \cdot \boldsymbol{n}$ on $\Sigma_{i}$ : the full corrective term, defined by $[10)$, which accounts for both the distal pressures contribution and the residual volume change of the cavity.

An investigation is first provided to evaluate the influence of the resistive term $R_{i}$ on the efficiency of the pressure correction. For this purpose, a case with no cavity motion (i.e., when $\left.\left.\boldsymbol{w}(t)\right|_{\partial \Omega_{2, \mathrm{D}}}=\boldsymbol{w}_{\mathbf{0}}(\boldsymbol{x}, t)\right)$ is considered and the term $g_{\Sigma_{i}, \mathrm{pc}}$ is used to correct the intracavity pressure. The set of values used for the resistive parameter $R_{i}$ are

$$
R_{i}=\left\{10^{i}, i=0 \ldots 10\right\} .
$$




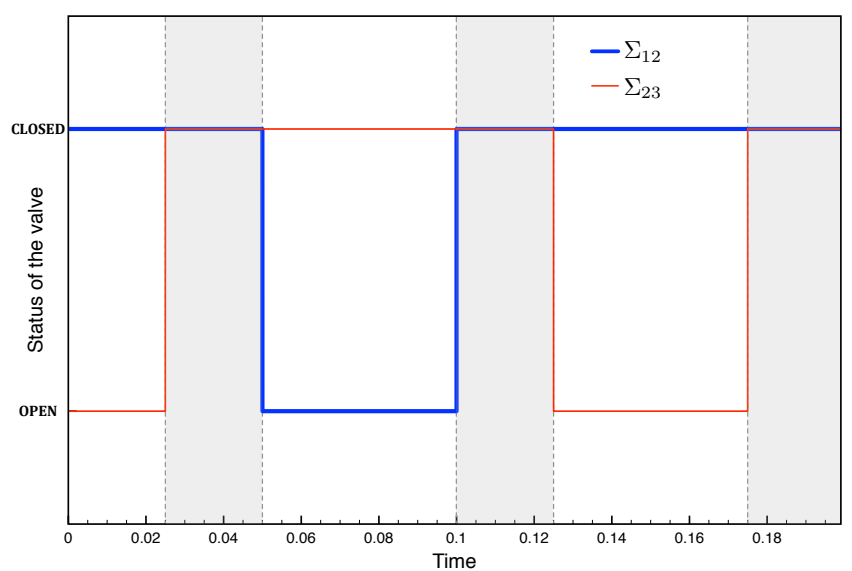

(a) Time history of the status of the valves.

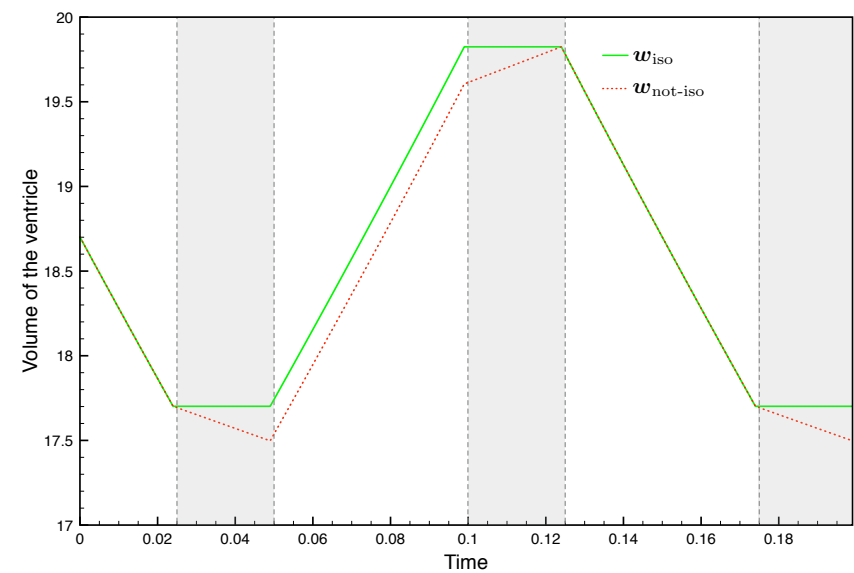

(b) Time history of the volume of the cavity $\Omega_{2}$.

FIGURE 7 Description of the isovolumetric phases considered for the toy problem model. The grey areas delimit the isovolumetric phases.

Defining $Q(t)$ as the inlet flow rate and $Q_{0}(t)$ as the inlet flow rate of a reference case in which no valve model is used, we define the logarithmic scaled residual flow rate $Q_{\text {res }}$ as:

$$
Q_{\text {res }}(t)=\log _{10}\left(\frac{Q(t)}{Q_{0}(t)}\right) .
$$

Its value is reported to quantify the actual flow reduction provided by the resistive immersed surfaces. For each value of $R_{i}$, the average pressure inside the cavity $\Omega_{2}$, denoted $\overline{P_{\Omega_{2}}}$, is compared with the reference pressure $P^{\star}$ to evaluate how good the intracavity pressure is corrected.

Following this first investigation, the best value of $R_{i}$ is then empirically fixed and used for all cases described in the following Table 1

\begin{tabular}{lccc}
\hline Case & $\left.\boldsymbol{w}\right|_{\partial \Omega_{2, \mathrm{D}}}$ & $g_{\Sigma_{12}}$ & $g_{\Sigma_{23}}$ \\
\hline$T_{1, \mathrm{NC}}$ & $\boldsymbol{w}_{0}(\boldsymbol{x}, t)$ & 0 & 0 \\
$T_{1, \mathrm{C}}$ & $\boldsymbol{w}_{0}(\boldsymbol{x}, t)$ & $g_{\Sigma_{12}, p c}$ & $g_{\Sigma_{23}, p c}$ \\
$T_{2, \mathrm{NC}}$ & $\boldsymbol{w}_{\text {iso }}(\boldsymbol{x}, t)$ & 0 & 0 \\
$T_{2, \mathrm{C}}$ & $\boldsymbol{w}_{\text {iso }}(\boldsymbol{x}, t)$ & $g_{\Sigma_{12}, p c}$ & $g_{\Sigma_{23}, p c}$ \\
$T_{3, \mathrm{NC}}$ & $\boldsymbol{w}_{\text {not-iso }}(\boldsymbol{x}, t)$ & 0 & 0 \\
$T_{3, \mathrm{PC}}$ & $\boldsymbol{w}_{\text {not-iso }}(\boldsymbol{x}, t)$ & $g_{\Sigma_{12}, p c}$ & $g_{\Sigma_{23}, p c}$ \\
$T_{3, \mathrm{C}}$ & $\boldsymbol{w}_{\text {not-iso }}(\boldsymbol{x}, t)$ & $g_{\Sigma_{12}, c}$ & $g_{\Sigma_{23}, c}$ \\
\hline
\end{tabular}

TABLE 1 Cases of pressure correction considered for the toy problem model.

Cases $T_{1}$ are designed in order to test the pressure correction in a simple, non-moving domain. Cases $T_{2}$ are designed to test the pressure correction in a more complex setting involving domain motion. Cases $T_{3}$ are designed to test the pressure correction in an even more challenging setting involving domain motion and breaking of the incompressibility constraint. The subscripts NC, PC and C denote, respectively, "No Correction", "Partial Correction", and "Correction". Note that for the cases $T_{1}$ and $T_{2}$, there is no distinction between partial correction and correction as the cavity is not undergoing volume change during the isovolumetric phases (i.e., when $\int_{\partial \Omega_{2}} \boldsymbol{w} \cdot \boldsymbol{n}=0$ ). The average pressure $\overline{P_{\Omega_{2}}}$ inside the cavity $\Omega_{2}$ is systematically computed in these different cases and compared with the prescribed pressure $P^{\star}$ to quantify the effectiveness of the pressure correction. 


\subsection{1 | Results}

\section{Sensitivity analysis of the resistance parameter}

Figure 8 depicts the evolution of $\left.Q_{\text {res }}(t)\right|_{t=0.17}$ with respect to the resistance value $R_{i}$. The time instant $t=0.17$ has been chosen to allow the flow rate $Q_{0}$ to be fully developed in the open channel. Moreover, it represents a time instant where only one valve of the toy problem model is closed, hence allowing to properly quantify the flow reduction of $Q$ compared to $Q_{0}$. It is noted that, as the resistance increases, the flow rate through the valves decreases, as expected from the standard RIS model. This result tends to justify the choice of a high value for the resistance $R_{i}$.

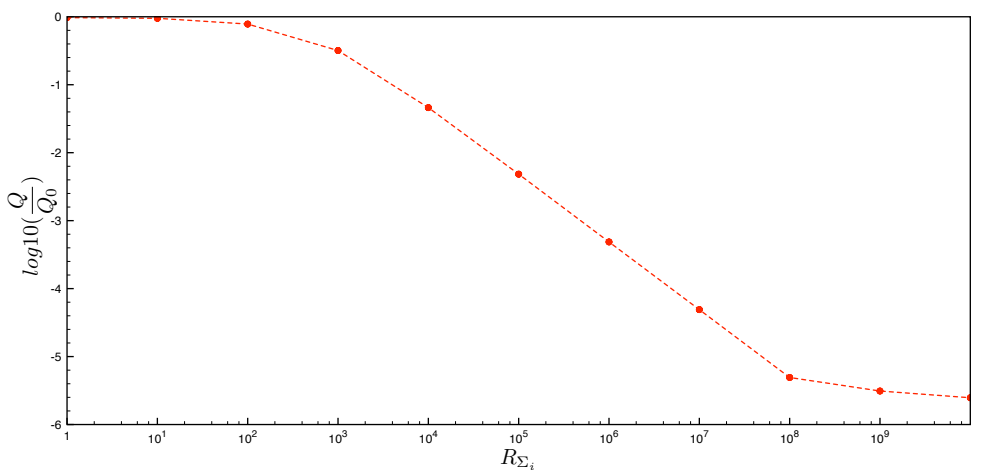

FIGURE 8 Log relative flow rate through the valves with respect to $R_{i}$ for the toy problem model.

On Figure 9. the pressures $\overline{P_{\Omega_{2}}}$ and $P^{\star}$ are compared, with respect to the time, using several values of $R_{i}$. From $R_{i}=10^{0}$ to $R_{i}=10^{6}$, the prescribed pressure $P^{\star}$ and computed pressure $\overline{P_{\Omega_{2}}}$ match properly during the isovolumetric phases depicting a successful behavior of the pressure correction. One can also notice that for $R_{i}=\left[10^{5}, 10^{6}\right]$, the RIS model is impermeable enough to also ensure proper pressure values outside the isovolumetric phases. For higher values of $R_{i}$, the pressure corrective term cannot ensure a good fit anymore. At $R_{i}=10^{7}$, a transition phase can be observed where $\overline{P_{\Omega_{2}}}$ is close to $P^{\star}$ but small steps are observed. At $R_{i}=10^{8}$ and beyond, the pressure correction is completely inefficient. This highlights the preference of a small value for the resistance $R_{i}$.

In conclusion, it is shown that a trade-off has to be made to ensure that the intracavity pressure is well corrected by the ARIS model while ensuring a good impermeability of the valve. In this specific setting, using $R_{i}=\left[10^{5}, 10^{6}\right]$ satisfies both conditions: the pressure inside $\Omega_{2}$ is properly corrected while ensuring a small residual flow rate compared with the open channel flow rate (i.e., $Q$ lower than $1 \%$ of $Q_{0}$ ). Based on this study, in the following, the value of the resistance $R_{i}$ is set to $10^{5}$.

Evaluation of the pressure correction for cases $T_{1}, T_{2}$ and $T_{3}$

Figures 10a 10b depict the results coming from, respectively, the cases $T_{1, \mathrm{NC}}$ and $T_{1, \mathrm{C}}$, for which no motion of the domain is imposed. In the case $T_{1, \mathrm{NC}}$, no pressure correction is applied. During the phases where both valves are closed, it is observed that the average pressure $\overline{P_{\Omega_{2}}}$ is approximately equal to $5 \times 10^{4}$. This result is coherent with the pressure estimation described by (4). Indeed, as $R_{12}=R_{23}$ and $\left|\Sigma_{12}\right|=\left|\Sigma_{23}\right|$, (4) can be rewritten as $P_{\Omega_{2}}=\frac{1}{2} P_{\Omega_{1}}+\frac{1}{2} P_{\Omega_{3}} \simeq 5 \times 10^{4}$, given that $P_{\Omega_{1}} \simeq 0$ and $P_{\Omega_{3}} \simeq 1 \times 10^{5}$ due to the considered applied Neumann normal boundary conditions. For the corrected case $T_{1, \mathrm{C}}(i . e$., when we are using the corrective term $g_{\Sigma_{i}, \mathrm{pc}}$ ), the intracavity pressure is now perfectly corrected during the isovolumetric phases.

Figures $11 \mathrm{a}-11 \mathrm{~b}$ depict the results coming from, respectively, the cases $T_{2, \mathrm{NC}}$ and $T_{2, \mathrm{C}}$. In those cases, a domain motion keeping the intracavity volume constant during the isovolumetric phases is imposed. Small peaks in the pressure are noticed during each transition between phases (e.g., $t=0.025$ or $t=0.05$ ) but these pressure peaks decay quickly toward stable values. Similar to the cases $T_{1}$, the average intracavity pressure is properly predicted by the theoretical analysis (4) in case $T_{2, \mathrm{NC}}$. For the case $T_{2, \mathrm{C}}$, using the corrective term $g_{\Sigma_{i}, \mathrm{pc}}$ allows to get the expected intracavity pressure during the isovolumetric phases.

Finally, Figures $12 \mathrm{a} / 12 \mathrm{c}$ depict the results coming from, respectively, the cases $T_{3, \mathrm{NC}}, T_{3, \mathrm{PC}}$ and $T_{3, \mathrm{C}}$. In these cases, an additional difficulty is addressed by considering a residual motion imposed during the isovolumetric phases. Compared to previously, a major difference is observed for the intracavity pressure for the non corrected case $T_{3, \mathrm{NC}}$. Indeed, the pressure is strongly offset 


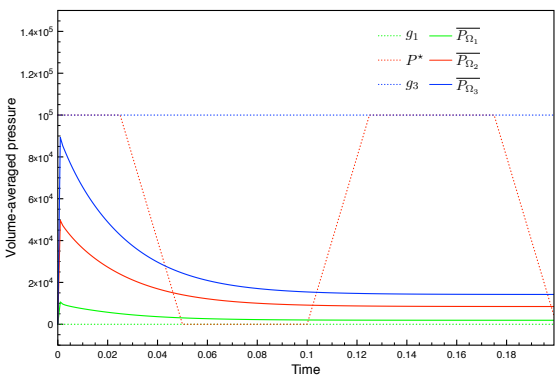

(a) $R_{i}=0$.

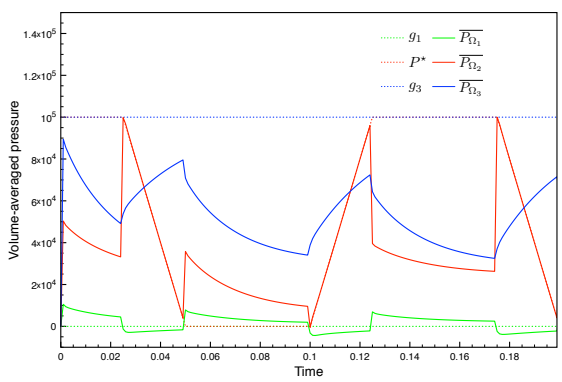

(d) $R_{i}=10^{2}$.

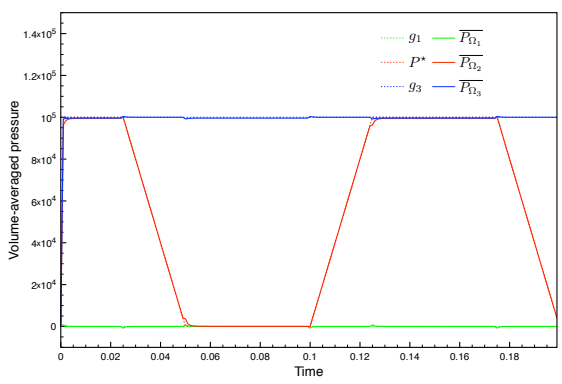

(g) $R_{i}=10^{5}$.

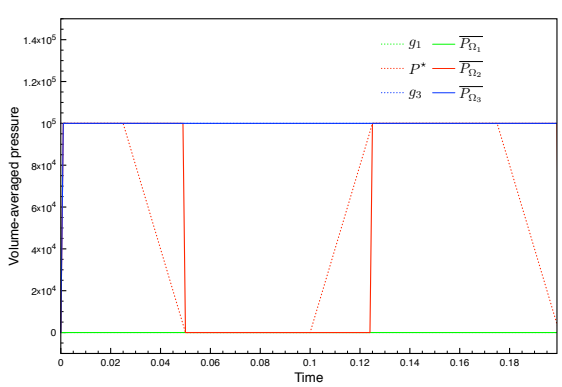

(j) $R_{i}=10^{8}$.

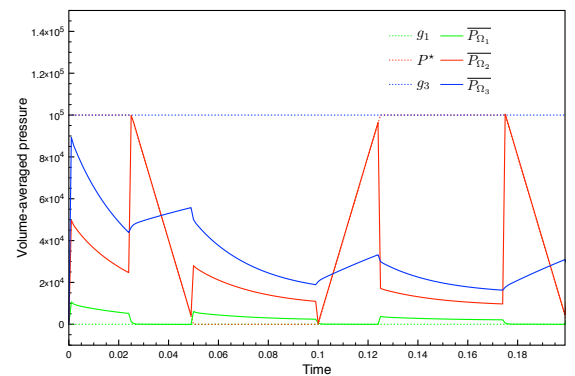

(b) $R_{i}=10^{0}$.

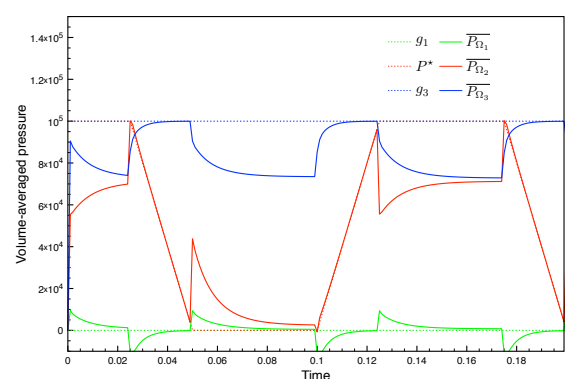

(e) $R_{i}=10^{3}$.

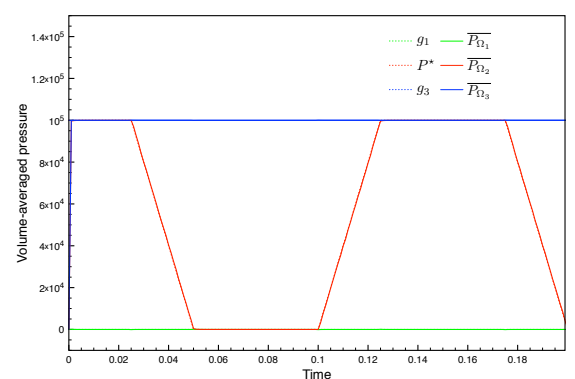

(h) $R_{i}=10^{6}$.

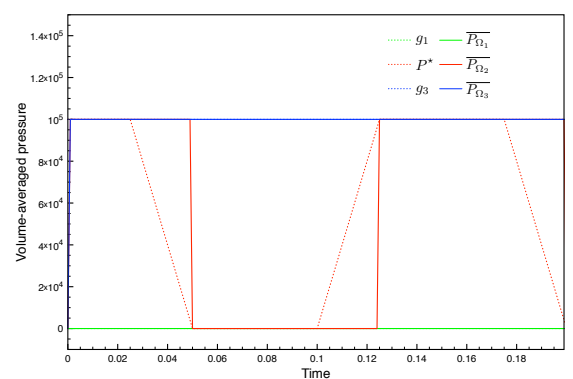

(k) $R_{i}=10^{9}$.

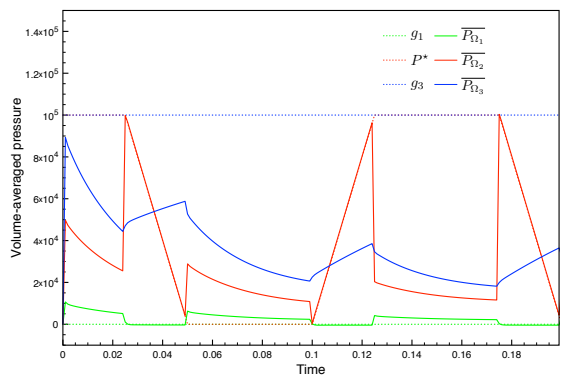

(c) $R_{i}=10^{1}$.

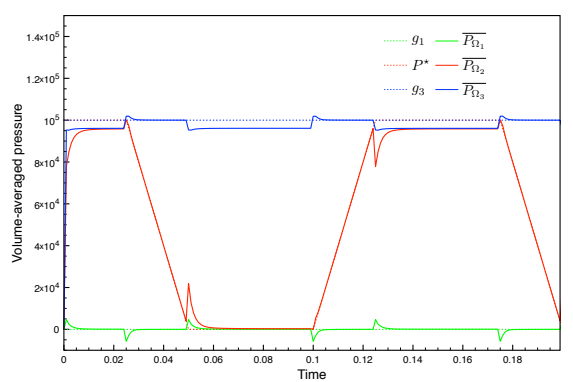

(f) $R_{i}=10^{4}$.

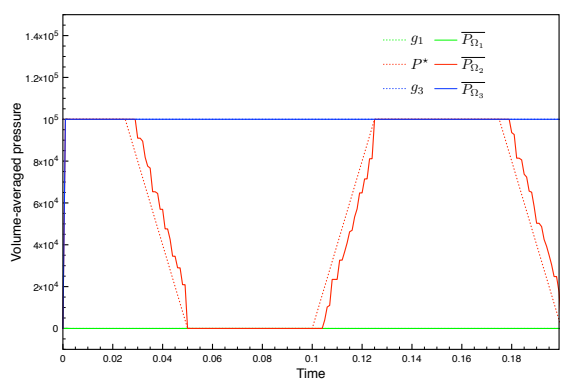

(i) $R_{i}=10^{7}$.

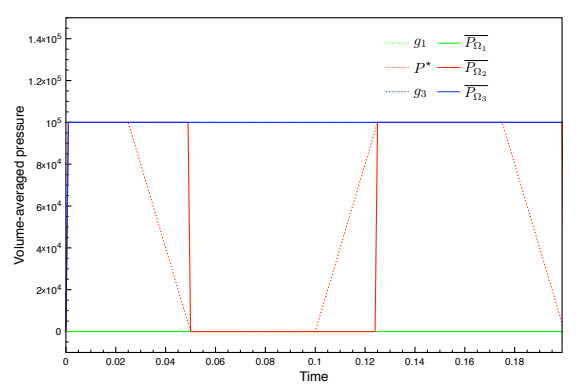

(I) $R_{i}=10^{10}$.

FIGURE 9 Time history of the computed pressures obtained with pressure correction for different values of $R_{i}$ for the toy problem model.

during the non-perfect isovolumetric phases: positively when the residual motion induces a contraction of the cavity and negatively when it induces a dilatation. This behavior has been predicted by the analysis provided in the discussion of Section 2.2 In the partially corrected case $T_{3, \mathrm{PC}}$, it may also be noted that the corrective term $g_{\Sigma_{i}, \mathrm{pc}}$ is not enough to correct the intracavity pressure as the residual motion of the cavity is not taken into account into the correction. On the opposite, using the complete corrective term $g_{\Sigma_{i}, \mathrm{c}}$, the intracavity pressure is corrected much more efficiently. In this case, it is observed that both the overall 


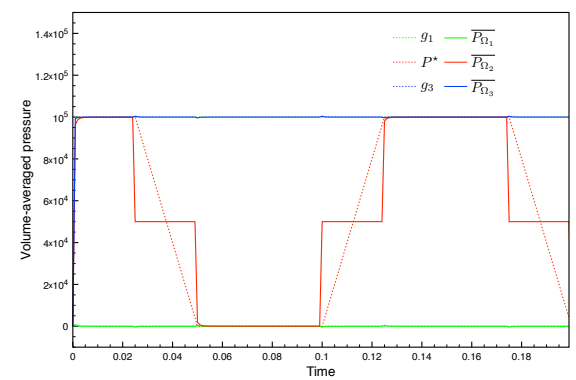

(a) Without correction.

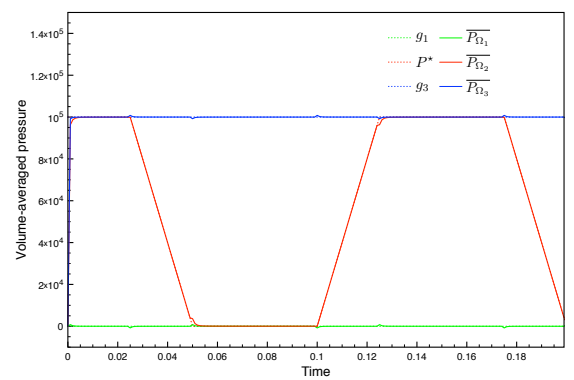

(b) With partial correction.

FIGURE 10 Time history of the computed pressures obtained for the cases $T_{1}$.

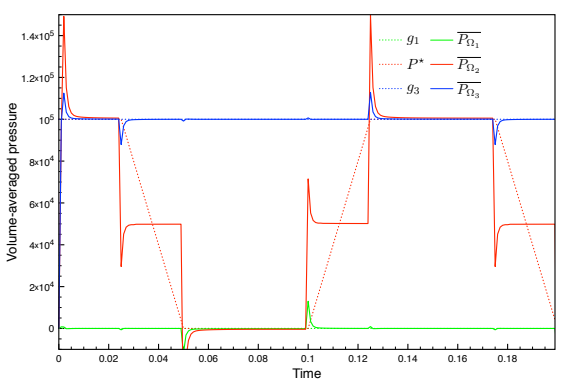

(a) Without correction.

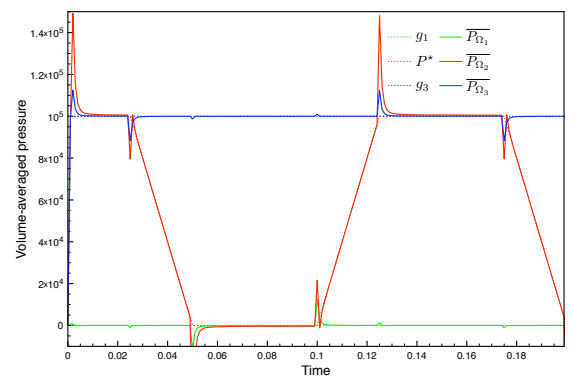

(b) With partial correction.

FIGURE 11 Time history of the computed pressures obtained for the cases $T_{2}$.

behavior of the intracavity pressure and its value match the prescribed pressure $P^{\star}$. Nevertheless, a small gap between the two pressure curves is observed, probably caused by the non-perfect validity of the simplifying assumptions (H1) and (H2).

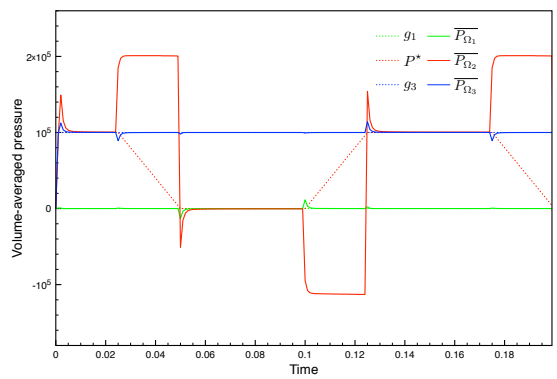

(a) Without correction.

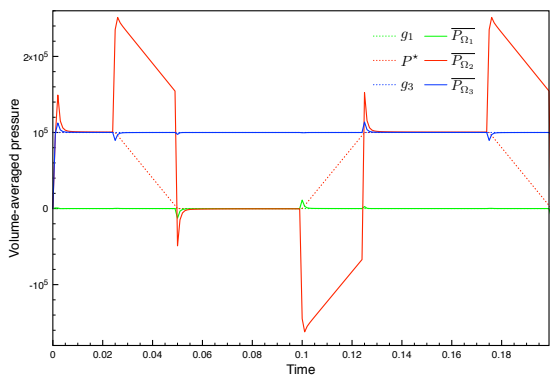

(b) With partial correction.

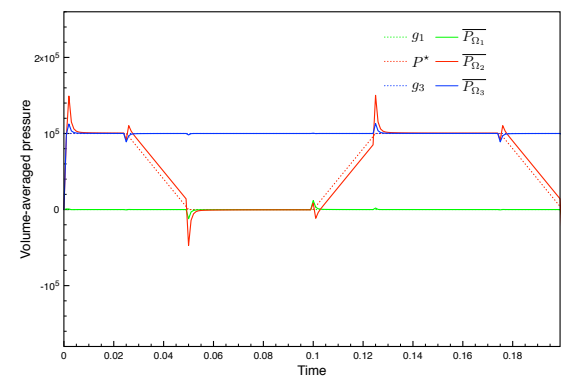

(c) With correction.

FIGURE 12 Time history of the computed pressures obtained for the cases $T_{3}$.

\section{2 | Realistic physiological model}

Since the pressure correction introduced in Section 2 has proved its benefits in correcting the intraventricular pressure of the toy problem model in several settings, we would like to apply it on a more physiological case. As the value of the intraventricular pressure is used to decide the status - active or inactive - of the resistive immersed surfaces ${ }^{5}$, its good evaluation is crucial to get the correct behavior of the valves. This is the main focus of the following numerical experiment on a physiological human heart geometry. 
We consider, for the fluid domain $\Omega$, a realistic portion of the human left heart, as depicted in Figure 13a For simplicity, we will use the physiological terminology of the cardiology to designate the different parts of the considered computational domain. Based on the previous notations of Section 2 the computational domains $\Omega_{1}, \Omega_{2}, \Omega_{3}$ correspond, respectively, to the portion of the atrium, to the full ventricle and to the portion of the aorta, as shown in Figure $13 \mathrm{~b}$ The typical dimensions of these computational domains are based on the physiological ones. All the units are given in the CGS units system. The different geometries come from a computerized tomography (CT) scan realized by the Zygote company. The immersed resistive valves, $\Sigma_{12}$ and $\Sigma_{23}$, correspond, respectively, to the mitral valve (see Figure 13c) and to the aortic valve (see Figure 13d). The former has been designed with the software 3-matic from physiological in vivo data ${ }^{13 / 14|15| 16 / 17]}$ whereas the latter is already included in the Zygote human heart model we use.

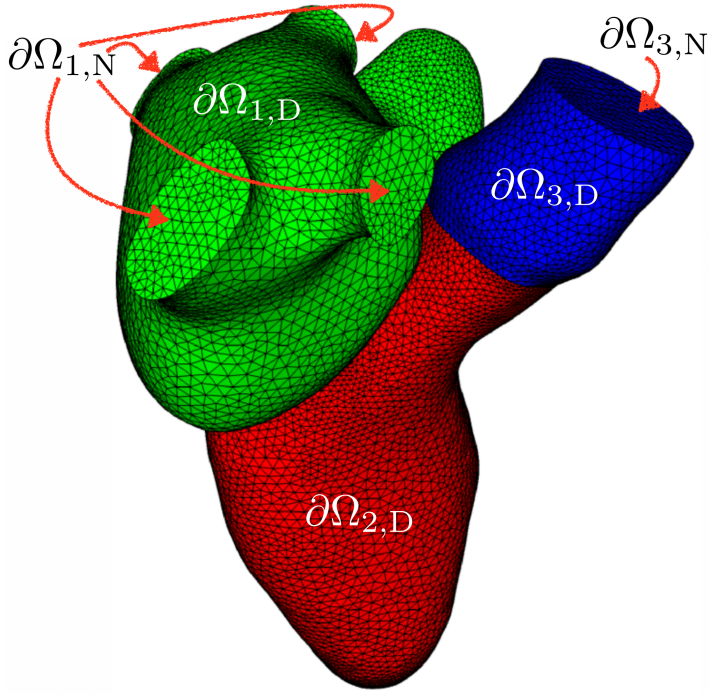

(a) Exterior of the fluid domain.

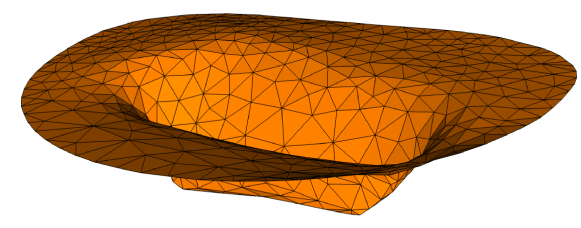

(c) Immersed surface $\Sigma_{12}$.

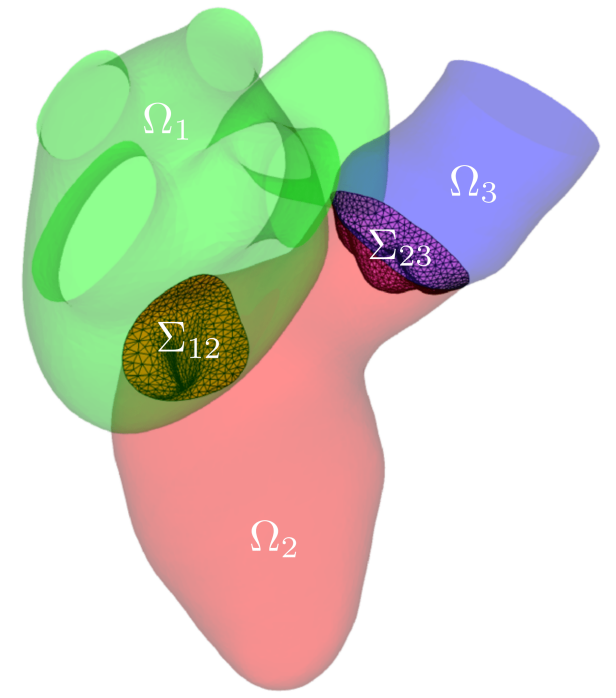

(b) Interior of the fluid domain.

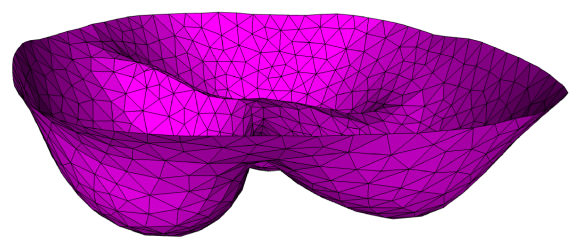

(d) Immersed surface $\Sigma_{23}$.

FIGURE 13 Computational domains used for the realistic model.

The physical parameters for the fluid are $\rho=1.06$ and $\mu=0.04$. The simulations are carried out for a total time of $t=0.85$, based on averaged physiological values for the heartbeat of a young adult $\frac{18}{18}$, with a time step $\tau=10^{-3}$. The fluid is initially at rest. The whole computational domain $\Omega$ is made of 326090 tetrahedron. The immersed surfaces, $\Sigma_{12}$ and $\Sigma_{23}$ are made of, respectively, 816 and 1474 triangular elements.

As regards the boundary conditions, a no-slip boundary condition is enforced on $\partial \Omega_{1, \mathrm{D}} \cup \partial \Omega_{2, \mathrm{D}} \cup \partial \Omega_{3, \mathrm{D}}$. The surface velocity $\left.\boldsymbol{w}\right|_{\partial \Omega_{2 \mathrm{D}}}$ is obtained from an electro-mechanical simulation ${ }^{19}$ in order to enhance the physiological validity of the considered numerical example. The pressure is prescribed on the inlet $\partial \Omega_{1, \mathrm{~N}}-$ corresponding to the cut ends of the pulmonary veins - as well as on the outlet $\partial \Omega_{3, \mathrm{~N}}$ - corresponding to the lower cut section of the aorta - using Neumann normal boundary conditions:

$$
\begin{array}{lll}
\boldsymbol{\sigma}(\boldsymbol{u}, p) \boldsymbol{n}=-g_{1} \boldsymbol{n} & \text { on } & \partial \Omega_{1}, \\
\boldsymbol{\sigma}(\boldsymbol{u}, p) \boldsymbol{n}=-g_{3} \boldsymbol{n} & \text { on } & \partial \Omega_{3} .
\end{array}
$$

The amplitude of these pressures, respectively denoted $g_{1}$ and $g_{3}$, are given by time-dependent functions coming from the above mentioned electro-mechanical model. Their values are depicted on Figure 14 In addition, the physiological ventricular 
pressure $P^{\star}$, also depicted in Figure 14 used in the corrective term 110 , comes from the same electro-mechanical simulation. Finally, a backflow stabilization based on a local regularization of the fluid velocity along the tangential directions on the Neumann boundaries ${ }^{20}$ is applied on $\partial \Omega_{1, \mathrm{~N}}$ and on $\partial \Omega_{3, \mathrm{~N}}$.

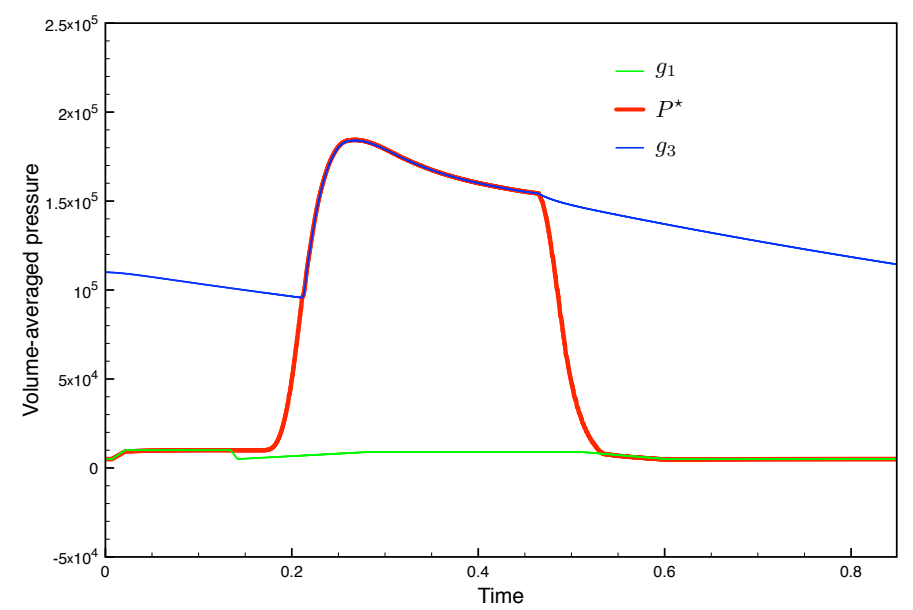

FIGURE 14 Time history of the reference pressures used for each cavity of the realistic model.

The surface displacement prescribed on $\partial \Omega_{2 . \mathrm{D}}$ is extended to the rest of domain using an appropriate non-linear lifting operator ${ }^{12}$ (denoted $\mathcal{L}$ in Section 2.1). Figures $15 \mathrm{a}$. $15 \mathrm{c}$ present some typical snapshots of the resulting displacement field, applied to the computational domain, respectively, at $t=0$ (initial state), $t=0.2$ (maximum contraction state) and $t=0.5$ (maximum dilatation state).

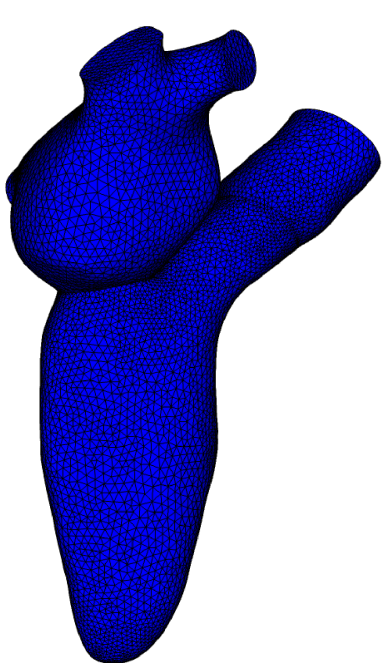

(a) $t=0$.

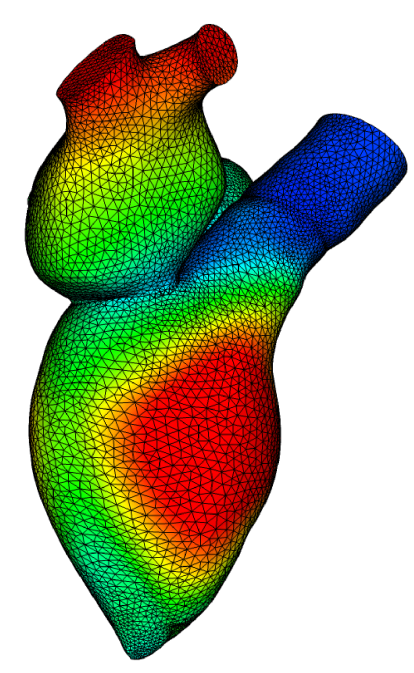

(b) $t=0.2$.

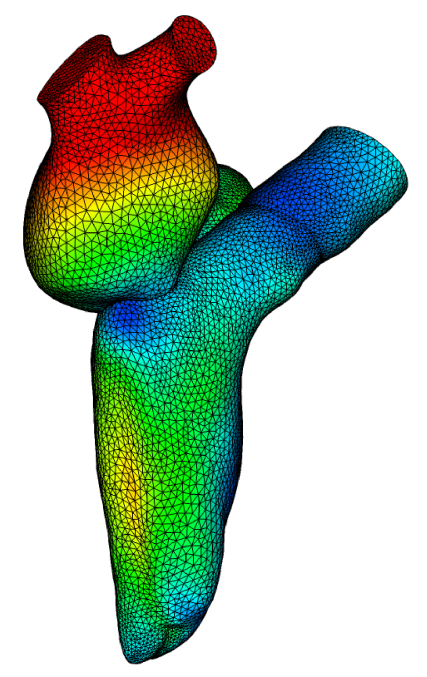

(c) $t=0.5$.

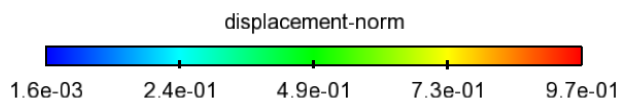

FIGURE 15 Snapshots of the displacement magnitude of the computational domain obtained for the realistic model.

In this realistic model, the values of $R_{12}$ and $R_{23}$ are no more arbitrarily prescribed but automatically handled by the physics of the problem, and more specifically by the local values of the velocity and pressure fields near the immersed surfaces. When 
a valve is closed, it will open if there is a positive pressure jump across it. On the opposite, when a valve is open, it will close if there is a backflow detected through its corresponding open surface. The corresponding physical decision criteria are depicted on Figure 16. For the immersed surface $\Sigma_{12}$, positive pressure jump and existing backflow mean, respectively, $p^{+}-p^{-}>0$ on $\Sigma_{12}$ and $\int_{\Sigma_{12}} \boldsymbol{u} \cdot \boldsymbol{n}^{+}<0$. For the immersed surface $\Sigma_{23}$, it means, respectively, $p^{-}-p^{+}>0$ on $\Sigma_{23}$ and $\int_{\Sigma_{23}} \boldsymbol{u} \cdot \boldsymbol{n}^{-}<0$. A refractory time, typically set to a few time steps, is introduced to prevent the valve from changing its status immediately after a first switch. This arbitrary constraint ensures a full development of the flow physics near the valve.

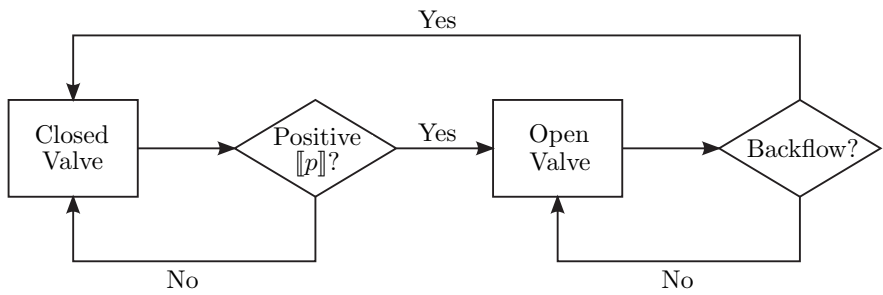

FIGURE 16 Flow chart representation of the resistive immersed surfaces for the realistic model.

Based on the pressure values previously depicted in Figure 14 two short isovolumetric phases (respectively defined by the time intervals $[t=0.135, t=0.215]$ and $[t=0.465, t=0.533])$ are then expected. The evolution of the volume of the cavity $\Omega_{2}$, given by the previous mentioned electro-mechanical simulation, is portrayed in Figure 17 As observed in real physiological cases 21 , the dilatation of the ventricle lasts longer than its contraction. Two zooms (the blue arrows) show that the incompressibility constraint is not perfectly verified during the isovolumetric phases. Therefore, the full pressure correction (12) is expected to be mandatory to compute the correct values of the pressures and hence the correct behavior of the resistive immersed valves.

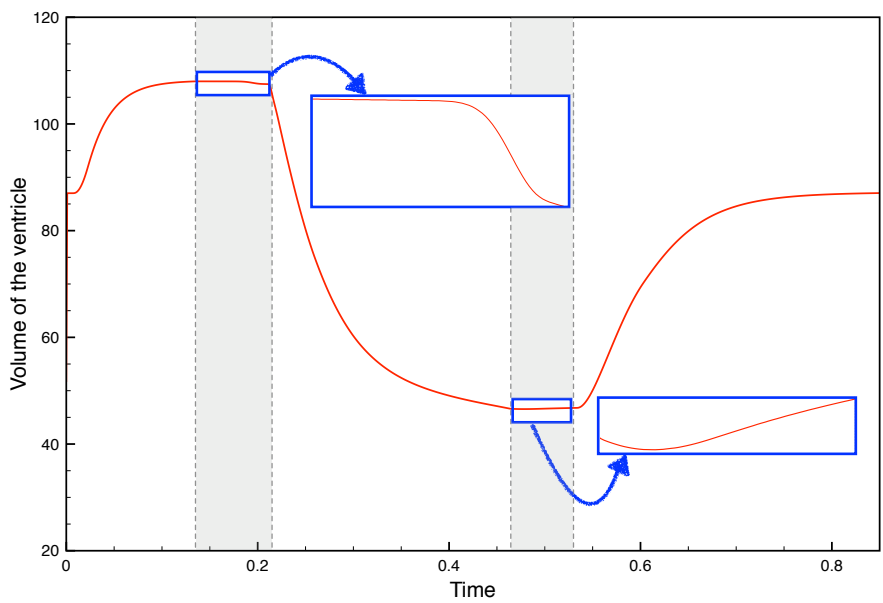

FIGURE 17 Time history of the volume of the ventricle for the realistic model. The grey areas delimit the expected isovolumetric phases.

In the following, the ARIS model is applied on this realistic model and the results obtained without pressure correction are compared with the ones obtained by using the corrective term given by $(10)$.

To assess the sensitivity of the efficiency of the pressure correction with respect to the parameter $R_{i}$ and to confirm our previous choice, the same analysis, as the one described in Section 3.1, is carried on here. The set of values used for the resistive parameter $R_{i}$ are still

$$
R_{i}=\left\{10^{i}, i=0 \ldots 10\right\} .
$$

Eventually, as the structure of the flow dynamics inside the ventricle can be used as a predictor of diseases or malfunction 226 , it is crucial to ensure that the new pressure correction does not perturb the velocity field. A comparative study of the velocity and 
pressure fields obtained during the first isovolumetric phase, with and without the pressure correction, is undertaken in order to highlight the absence of disruption. The first phase is the most pertinent for this comparison as its correct computation is crucial and mandatory to not perturb the physics of the developed flow and, hence, the next time steps of the simulation.

\subsection{1 | Results}

On Figure 18 the matching of the evolution of the pressures $\overline{P_{\Omega_{2}}}$ and $P^{\star}$, obtained with the pressure correction, are compared for several values of $R_{i}$ with respect to the time. The goal is to determine the optimal value of the resistive parameter.

Firstly, positive and negative peak values are observed for $\overline{P_{\Omega_{2}}}$, respectively, at $t \simeq 0.21$ and at $t \simeq 0.54$ (i.e., respectively, just after the first and the second isovolumetric phases), on all cases. This behavior is similar to what has been observed for the toy model in Section 3.1.1 Moreover, even for a case where the pressure correction is not applied (i.e., when $R_{i}=0$ ), the same pressure peaks are obtained, as depicted on Figure 18a This highlights the fact that these local quickly decaying extrema for $\overline{P_{\Omega_{2}}}$ are not induced by the introduced pressure correction. A possible explanation would be that the resistance changes, due to valve opening and closing, are explicitly imposed to the fluid. As a result, some time steps are required for the fluid to adapt to these topological changes, inducing pressure oscillations. We refer the reader to the conclusion for some perspectives on how to tackle this behavior.

Figure $18 \mathrm{a}$ depicts the case where no immersed valve is used (as $R_{i}$ is set to 0 ) and where the matching of the pressures is hence, obviously, impossible to obtain. The computed pressure $\overline{P_{\Omega_{2}}}$ of Figures $18 \mathrm{~b} 18 \mathrm{f}$ fail to match the reference $P^{\star}$ outside the isovolumetric phases, due to the bad behavior of the immersed valves which keep fluctuating quickly between their closed and open configurations. This phenomenon is illustrated by the pressures oscillations. Among all the cases, the best pressures matching is obtained for $R_{i}=10^{5}$, as observed on Figure $18 \mathrm{~g}$. When the resistance is increased beyond this optimal value, results start to worsen, especially for the first isovolumetric phase, as depicted on Figures $18 \mathrm{~h}-18 \mathrm{j}$. For the case $R_{i}=10^{6}$, portrayed on Figure $18 \mathrm{~h}$ even if $\overline{P_{\Omega_{2}}}$ is confined between $\overline{P_{\Omega_{1}}}$ and $\overline{P_{\Omega_{3}}}$, it is not monotonous, as it should be in a physiological case. For the two following cases, due to the inaccurate computed physics, and more precisely the wrong intraventricular pressure, the valve status change several time during the isovolumetric phases while they were supposed to keep being closed. Beyond the case of $R_{i}=10^{8}$, where $\overline{P_{\Omega_{2}}}$ present very strong oscillations for $t \in[0.135,0.215]$ and for $t \in[0.465,0.533]$, as represented on Figure 18j the other cases fail to converge and the simulations stops around $t=0.2$, as portrayed on Figures $18 \mathrm{k}$ - 181 Based on this study, in the following, the value of the resistance $R_{i}$ is now set to $10^{5}$.

Figures $19 \mathrm{a}-19 \mathrm{~b}$ depict the pressure curves coming from, respectively, the case without and with the use of the corrective term (10), both for the optimized value of $R_{i}=10^{5}$. It must be noted that, except for the isovolumetric phases, the computed pressures between both cases are almost perfectly identical. This underlines the fact that the corrective term, only used during the isovolumetric phases, does not have an impact on the other instants of the simulation, as expected.

As portrayed in Figure $19 \mathrm{a}$, for the case without correction, $\overline{P_{\Omega_{2}}}$ is not well-defined when both valves are closed and get arbitrary values. Therefore, this induces wrong behaviors of the immersed valves. On the opposite, when the corrective term is applied, $\overline{P_{\Omega_{2}}}$ is now correctly defined and the computed pressures match the reference ones, as highlighted in Figure $19 \mathrm{~b}$

The time history of the valves status of each immersed surface $\Sigma_{12}$ and $\Sigma_{23}$ obtained for the cases without and with pressure correction are depicted, respectively, on Figures 20a 20b. Major differences are observed during the first isovolumetric phase: non physiological changes of configuration of each valve are obtained when no pressure correction is applied while the expected correct behavior, similar to the one used for the toy model on Figure $7 \mathrm{a}$ is get with the pressure correction. This difference of behavior is crucial to correct as a wrong isovolumetric phase will induce a erroneous computation of the flow characteristics during the rest of the simulation.

Figures $21 \mathrm{a} 21 \mathrm{~b}$ present the snapshots of the velocity field obtained during the middle of the first isovolumetric phase, at $t=0.185$, respectively, for the case without and with pressure correction. The same typical features of the velocity field, especially its vorticity, are obtained for the two cases, illustrating the fact that the additional normal stress term (8), applied on the immersed surfaces, keeps the velocity field comparable.

Snapshots of the pressure fields obtained without and with optimized pressure correction are given in Figures 22a 22b respectively. On both figures, observation of the ventricular cavity reveals that even though $P_{\Omega_{2}}$ is not perfectly homogeneous on the scale of the ventricle - as it is assumed in (H2) - the pressure values near the first immersed surface $\Sigma_{12}$ and near the second immersed surface $\Sigma_{23}$ are approximately equal. This observation highlights the fact that using the same value $P_{\Omega_{2}}$ for the negative sided-restrictions of the pressure $p^{-}$in equations (5) and (6), respectively, for both immersed surfaces $\Sigma_{12}$ and $\Sigma_{23}$, is coherent. 


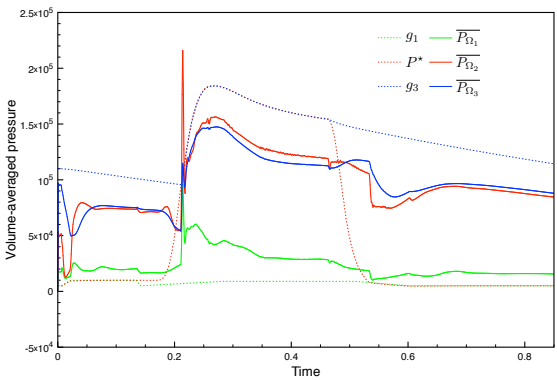

(a) $R_{i}=0$.

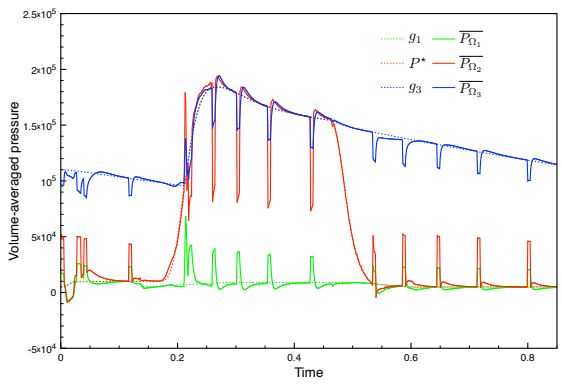

(d) $R_{i}=10^{2}$.

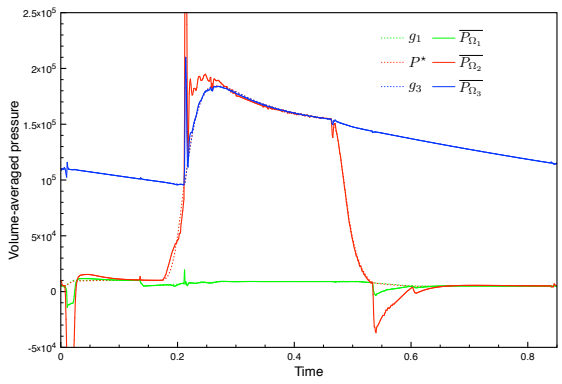

(g) $R_{i}=10^{5}$.

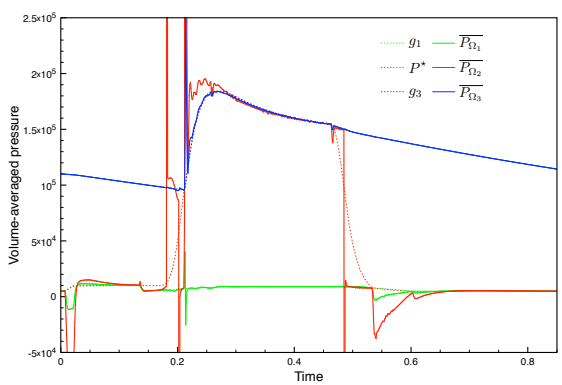

(j) $R_{i}=10^{8}$.

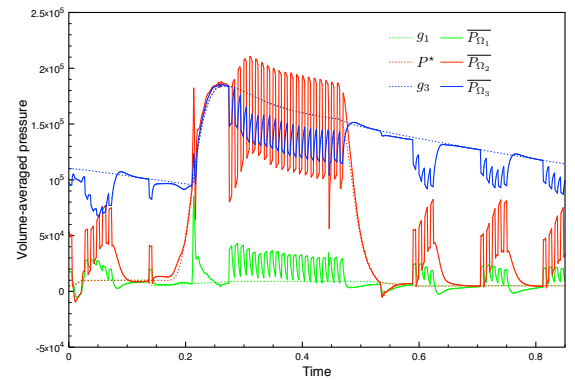

(b) $R_{i}=10^{0}$.

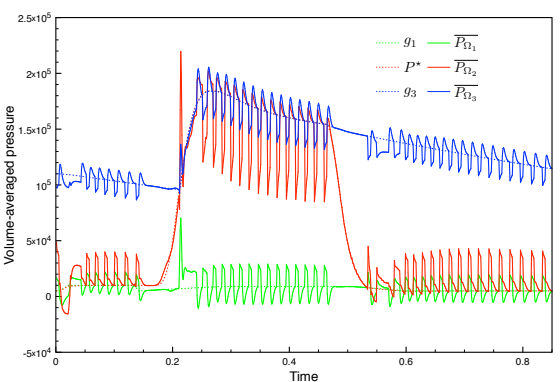

(e) $R_{i}=10^{3}$.

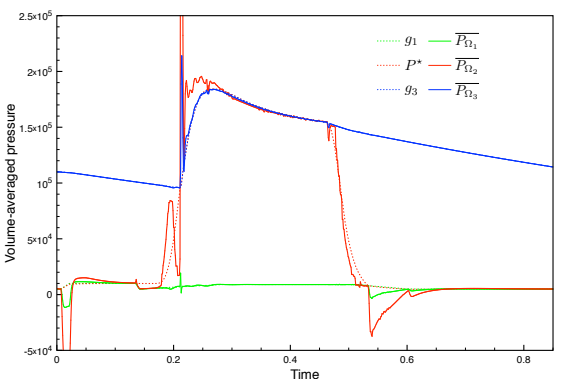

(h) $R_{i}=10^{6}$.

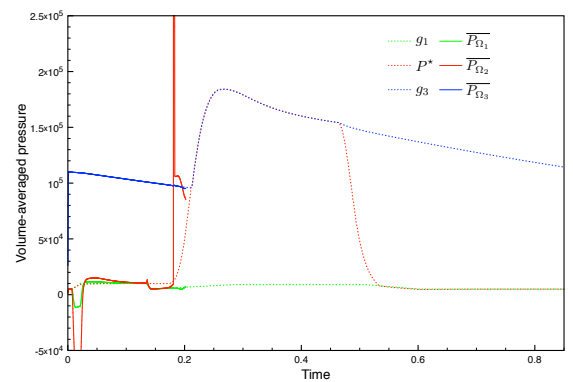

(k) $R_{i}=10^{9}$.

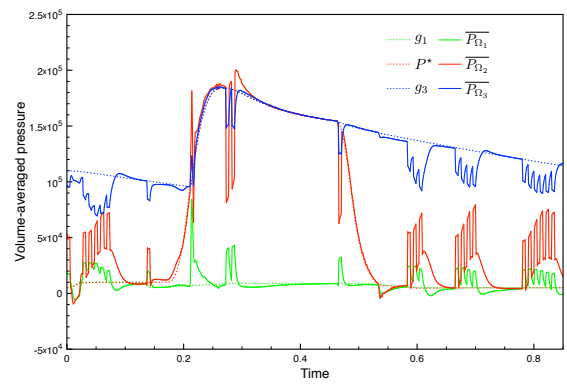

(c) $R_{i}=10^{1}$.

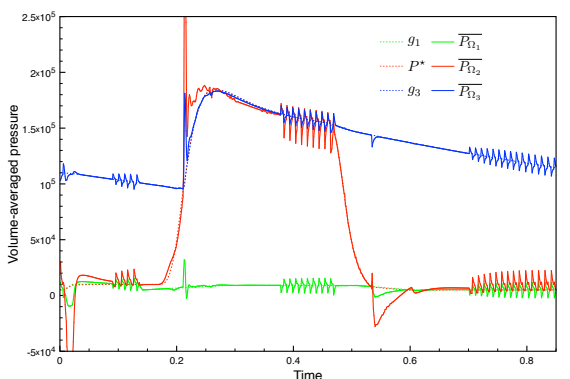

(f) $R_{i}=10^{4}$.

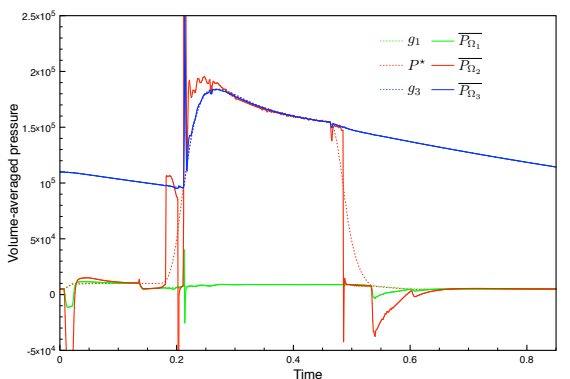

(i) $R_{i}=10^{7}$.

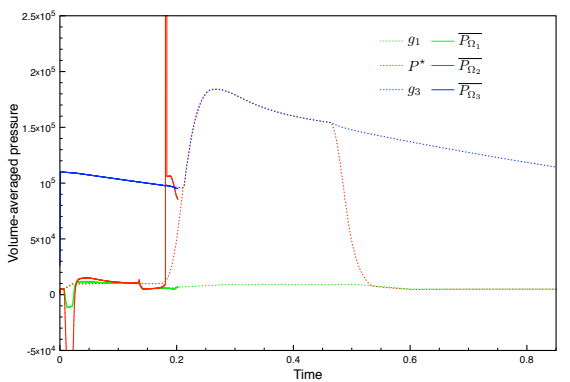

(I) $R_{i}=10^{10}$.

FIGURE 18 Time history of the computed pressures obtained with pressure correction for different values of $R_{i}$ for the realistic model.

Based on the reference pressures of Figure $\overline{14}, \overline{P_{\Omega_{2}}}$ is expected, at $t=0.185$, to belong to the interval of values $\left[\overline{P_{\Omega_{1}}}, \overline{P_{\Omega_{3}}}\right]$. The intraventricular pressure is correctly computed when pressure correction is applied whereas, when there is no correction, its value is overestimated and even exceed the aortic one, as illustrated on Figure 22a. This highlights the benefits brought by the pressure corrective term (10) concerning the computation of the correct intermediate values for the pressure during the isovolumetric phases. 


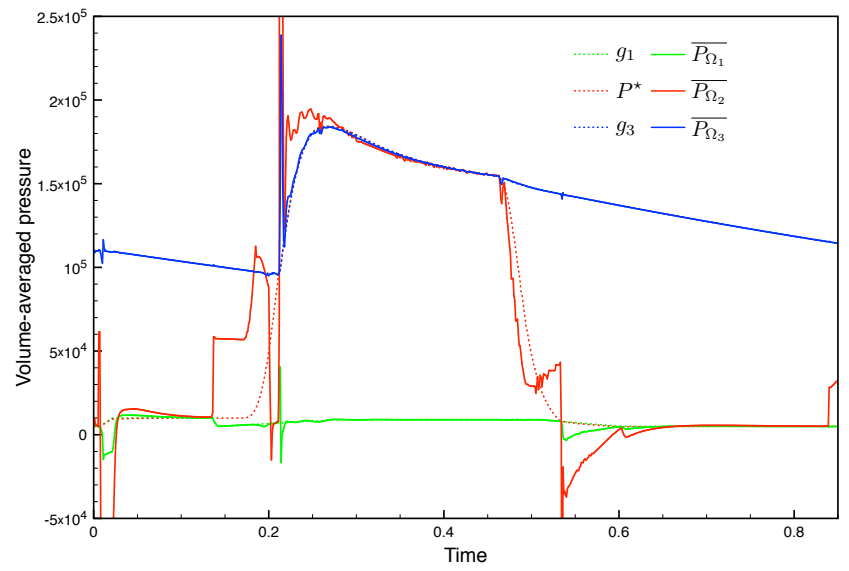

(a) Without correction.

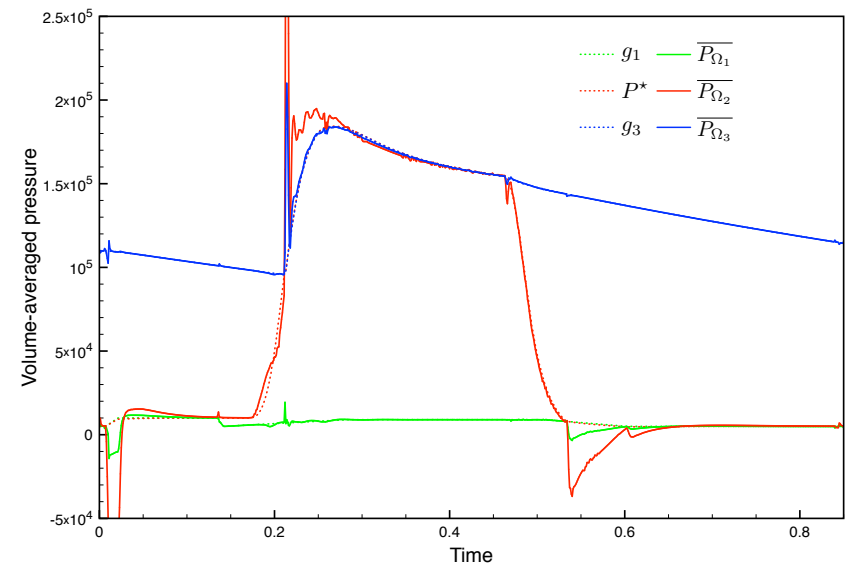

(b) With correction.

FIGURE 19 Time history of the computed pressures for the optimized value of $R_{i}=10^{5}$ for the realistic model.

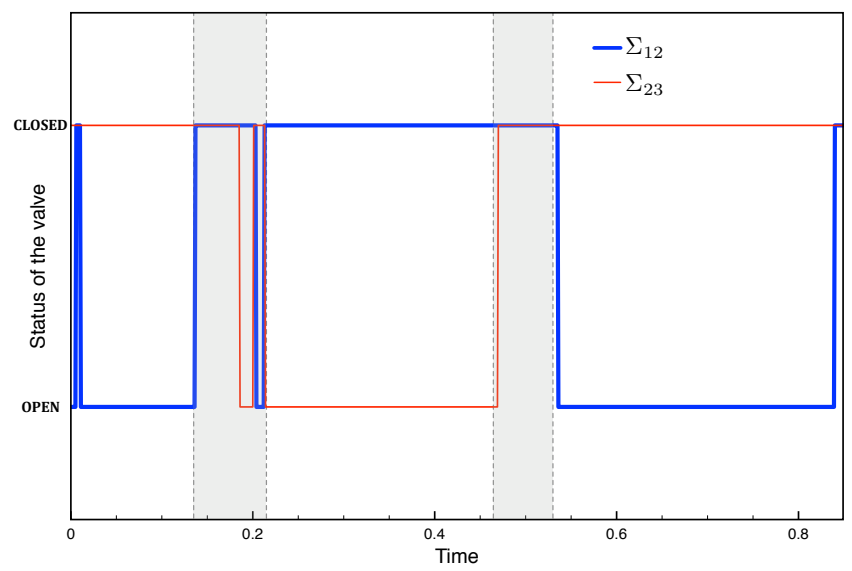

(a) Without correction.

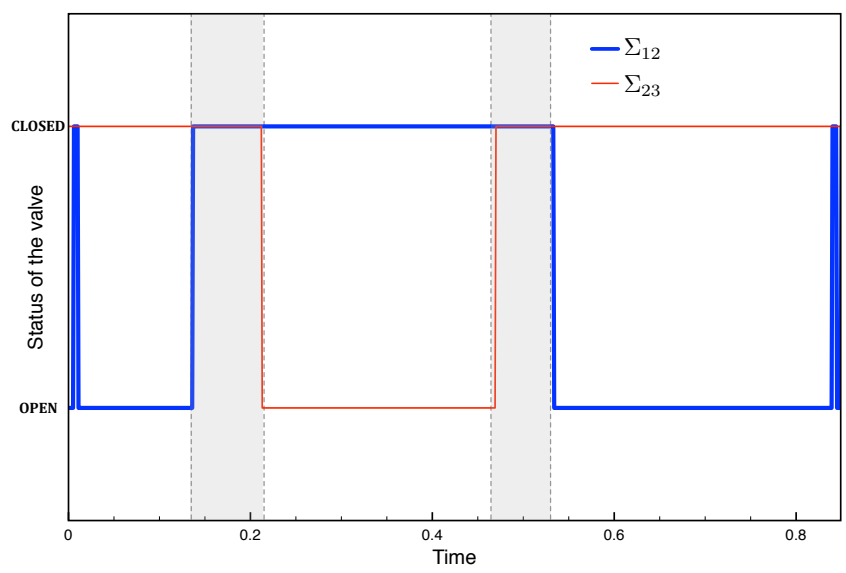

(b) With correction.

FIGURE 20 Time history of the status of the valves obtained for the realistic model. The grey areas delimit the expected isovolumetric phases. 


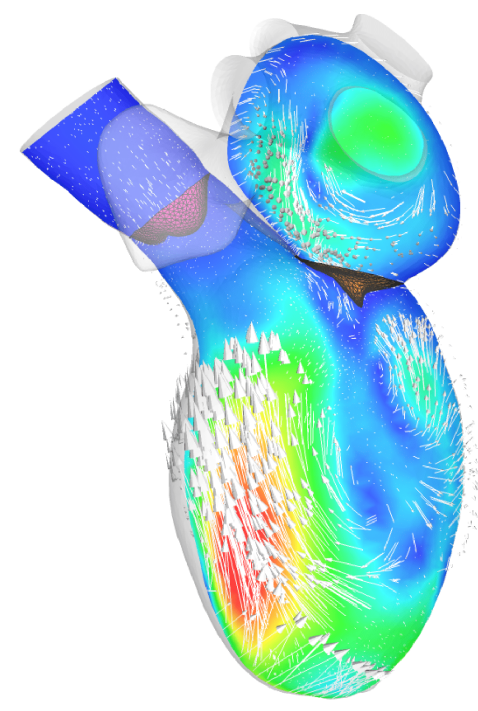

(a) Without correction.

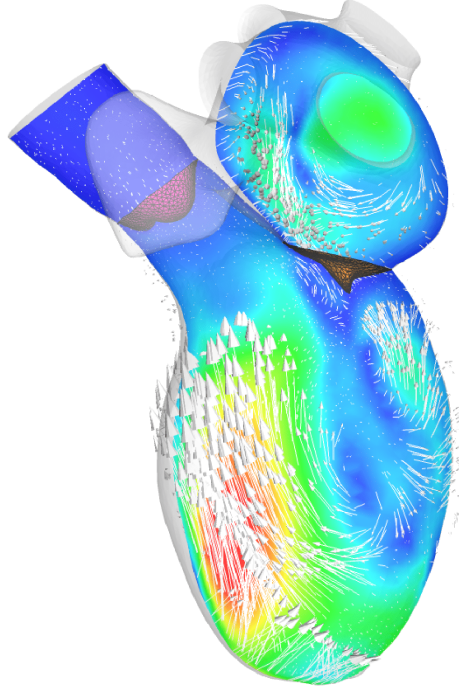

(b) With correction.

\begin{tabular}{ccccc}
\multicolumn{5}{c}{ velocity-norm } \\
\hline 0 & 12 & 25 & 38 & 50
\end{tabular}

FIGURE 21 Snapshots of the velocity field obtained at $t=0.185$ for the realistic model.

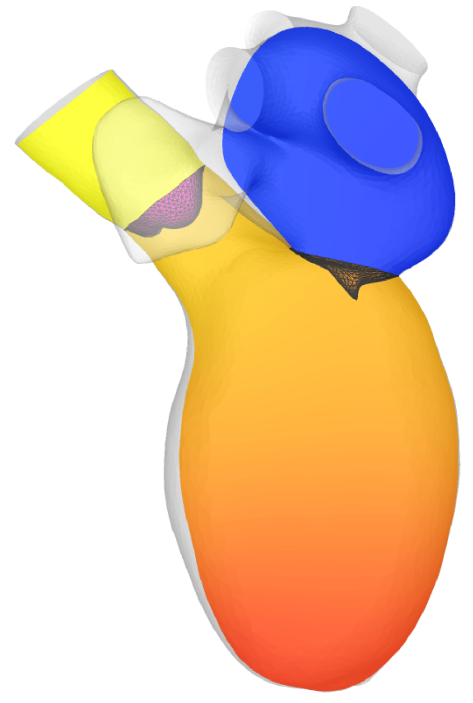

(a) Without correction.

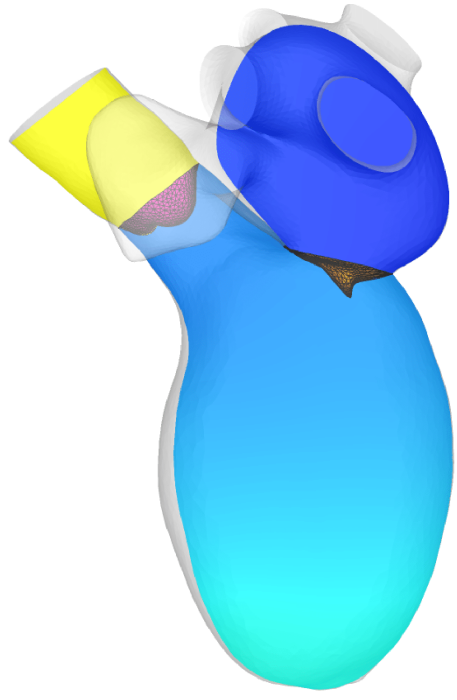

(b) With correction.

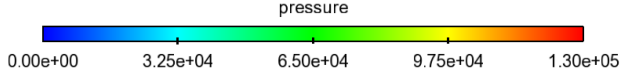

FIGURE 22 Snapshots of the pressure field obtained at $t=0.185$ for the realistic model. 


\section{4 | CONCLUSIONS}

In this paper, a new reduced model for heart valves has been introduced for the description of the isovolumetric phases within an uncoupling approach.

In Section 2.1. the original RIS mode ${ }^{955}$ has been recalled and its application to heart valves modeling has been presented. The generic problem of a fluid cavity enclosed by two closed RIS valves has been considered by providing an estimate of the internal pressure in Section 2.2 A simplified expression estimating the pressure of the cavity has been derived, highlighting that its value depends on several parameters including the exterior pressures, the resistive term of the RIS model and the wall cavity velocities. This analysis proved why realistic isovolumetric phases could not be obtained with the standard RIS model. In Section 2.3, the new reduced model, called ARIS, has been presented. An additional normal stress term was introduced in order to counteract the contributions of the exterior pressures and wall velocities.

In Section 3, two numerical examples have been considered to illustrate the benefits of this new approach. First, a toy model with a simplified geometry has been designed to reproduce the main characteristics of more complex heart simulations. Arbitrary pressure values and several different domain motions have been prescribed to test the efficiency of the ARIS model in different configurations. The second numerical example has consisted of a physiological left heart geometry where the pressure values and domain motions were provided by an external electro-mechanical simulation. In all those cases, the original RIS model failed to properly model the intracavity pressures and the valves behavior during the isovolumetric phases. These experiments highlighted that the intracavity pressure could be computed using the pressure estimate described by (4). It also highlighted that the violation of the incompressibility constraint and its correction is a key challenge to compute the correct intraventricular pressure during the isovolumetric phases.

In both numerical examples, an analysis of the influence of the valve resistive parameter $R_{i}$ showed that a trade-off had to be made between a proper correction of the intracavity pressure and an acceptable impermeability of the valves. Indeed, the introduced corrective term benefits from the fact that the RIS model is penalizing the fluid on the immersed surfaces and is only enforcing Dirichlet boundary conditions in a weak sense. However, as the resistive term keeps increasing, the RIS model converges toward a singular problem equivalent to imposing only Dirichlet boundary conditions. In this setting, the pressure correction was not working anymore.

The optimum value of $R_{i}$ has been determined in each example and its use in the ARIS model led to strong improvements in the physiological validity of the numerical simulations compared to the traditional RIS model. In the cardiac test case, it was shown that the ARIS model was able to control the left ventricular pressure during the isovolumetric phases resulting in a better physiological behavior of the valves. Moreover, the ARIS model properly corrected the intracavity pressure without alteration of the velocity field and its main features.

The proposed approach therefore offers a good compromise between standard uncoupling and complex FSI models. By expanding the original RIS formulation with an additional reference value, the physiological behavior of the intracavity pressure during the isovolumetric phases is partially recovered without using fully coupled fluid-structure models. Nevertheless, one limitation of applying the ARIS formulation is the potential difficulty to obtain such reference data. In particular, when aiming to produce patient-specific simulations, recovery of the intraventricular pressure by catheterization is an invasive procedure and might not always be possible. Moreover, it must also be reminded that the simplifications introduced by using this reduced model might affect and disrupt the intraventricular flow. As mentioned in Section 3.2.1. some pressure oscillations were observed due to the explicit treatment of the reduced valve model. A first approach to circumvent this issue could to formulate the valve dynamics as a non-linear constraint on the fluid velocity. Another interesting extension of this work could be to compare the results coming from the ARIS model with the ones coming from a fully coupled FSI simulation in order to quantify the potential inaccuracies induced by the current simplified approach.

\section{ACKNOWLEDGEMENTS}

The authors wish to thank D. Chapelle, P. Moireau, R. Chabiniok, G. Bureau and colleagues, from the M3DISIM Inria projectteam, for providing data on displacements and pressure of the left ventricle, used in Section 3.2 and for their support with the mechanical simulations. The research has been partially supported by a CIFRE Ph.D convention established between INRIA Paris and Philips Research, France. The research leading to these results has also been partially supported by the project MIVANA. MIVANA is a collaborative project for the development of new technologies for mitral valve repair. It is led by the 
start-up company Kephalios, with the participation of the start-up company Epygon, and it has received funds from the French government, in the context of the program "Investissement d'Avenir".

\section{References}

1. Lai M, Peskin C. An immersed boundary method with formal second-order accuracy and reduced numerical viscosity. $J$. Comp. Phys. 2000; 160(2): 705-719. doi: 10.1006/jcph.2000.6483

2. Loon vR, Anderson P, Hart dJ, Baaijens F. A combined fictitious domain/adaptive meshing method for fluid-structure interaction in heart valves. Int. J. Num. Meth. Fluids 2004; 46(5): 533-544. doi: 10.1002/fld.775

3. Kamensky D, Hsu MC, Schillinger D, et al. An immersogeometric variational framework for fluid-structure interaction: application to bioprosthetic heart valves. Comput. Methods Appl. Mech. Eng. 2015; 284: 1005-1053. doi: 10.1016/j.cma.2014.10.040

4. Gao H, Feng L, Qi N, Berry C, Griffith B, Luo X. A coupled mitral valve-left ventricle model with fluid-structure interaction. Med. Eng. Phys. 2017; 47: 128-136. doi: 10.1016/j.medengphy.2017.06.042

5. Astorino M, Hamers J, Shadden SC, Gerbeau JF. A robust and efficient valve model based on resistive immersed surfaces. Int. J. Numer. Meth. Biomed. Engng. 2012; 28(9): 937-959. doi: 10.1002/fld.775

6. Chnafa C, Mendez S, Nicoud F. Image-Based Simulations Show Important Flow Fluctuations in a Normal Left Ventricle: What Could be the Implications?. Ann. Biomed. Eng. 2016; 44(11): 3346-3358. doi: 10.1007/s10439-016-1614-6

7. Fedele M, Faggiano E, Dedè L, Quarteroni A. A patient-specific aortic valve model based on moving resistive immersed implicit surfaces. Biomech. Model. Mechanobiol. 2017; 16(5): 1779-1803. doi: 10.1007/s10237-017-0919-1

8. Tagliabue A, Dedè L, Quarteroni A. Fluid dynamics of an idealized left ventricle: the extended Nitsche's method for the treatment of heart valves as mixed time varying boundary conditions. Int. J. Num. Meth. Fluids 2017; 85(3): 135-164. doi: $10.1002 /$ fld. 4375

9. Fernández M, Gerbeau JF, Martin V. Numerical simulation of blood flows through a porous interface. M2AN Math. Model. Numer. Anal. 2008; 42(6): 961-990. doi: 10.1051/m2an:2008031

10. Caiazzo A, Fernández M, Gerbeau JF, Martin V. Projection Schemes for Fluid Flows through a Porous Interface. SIAM Jour. on Sc. Comp. 2011; 33(2): 541-564. doi: 10.1137/100788124

11. Fukuta H, Little W. The Cardiac Cycle and the Physiological Basis of Left Ventricular Contraction, Ejection, Relaxation, and Filling. Heart Failure Clin. 2008; 4(1): 1-11. doi: 10.1016/j.hfc.2007.10.004

12. Landajuela M, Vidrascu M, Chapelle D, Fernández M. Coupling schemes for the FSI forward prediction challenge: comparative study and validation. Int. J. Numer. Meth. Biomed. Engng. 2017; 33(4): e2813. doi: 10.1002/cnm.2813

13. Ranganathan N, Lam J, Wigle E, Silver M. Morphology of the Human Mitral Valve: II. The Valve Leaflets. Circulation 1970; 41(3): 459-467. doi: 10.1161/circ.41.3.459

14. Rusted I, Scheifley C, Edwards J. Studies of the mitral valve. I. Anatomic features of the normal mitral valve and associated structures. Circ. 1952; 6(6): 825-831. doi: 10.1161/01.CIR.6.6.825

15. Du Plessis L, Marchand P. The anatomy of the mitral valve and its associated structures. Thorax 1964; 19: 221-227. doi: 10.1136/thx.19.3.221

16. Naoum C, Leipsic J, Cheung A, et al. Mitral Annular Dimensions and Geometry in Patients With Functional Mitral Regurgitation and Mitral Valve Prolapse. JACC: Card. Imag. 2016; 9(3): 269-280. doi: 10.1016/j.jcmg.2015.08.022

17. Votta E, Caiani E, Veronesi F, Soncini M, Montevecchi F, Redaelli A. Mitral valve finite-element modelling from ultrasound data: a pilot study for a new approach to understand mitral function and clinical scenarios. Phil. Trans. A Math. Phys. Eng. Sci. 2008; 366(1879): 3411-34. doi: 10.1098/rsta.2008.0095 
18. Hart J. Normal resting pulse rate ranges. J. Nurs. Educ. Pract. 2015; 5(8). doi: 10.5430/jnep.v5n8p95

19. Chapelle D, Le Tallec P, Moireau P, Sorine M. Energy-Preserving Muscle Tissue Model: Formulation and Compatible Discretizations. Int. J. for Multi. Comp. Engng. 2012; 10(2): 189-211. doi: 10.1615/IntJMultCompEng.2011002360

20. Esmaily Moghadam M, Bazilevs Y, Hsia TY, Vignon-Clementel I, Marsden A, Congenital Hearts Alliance (MOCHA) oM. A comparison of outlet boundary treatments for prevention of backflow divergence with relevance to blood flow simulations. Comput. Mech. 2011; 48(3): 277-291.

21. Wikstrand J, Berglund G, Wilhelmsen L, Wallentin I. Value of systolic and diastolic time intervals. Studies in normotensive and hypertensive 50-year-old men and in patients after myocardial infarction. Br. Heart J. 1978; 40(3): 256-267. doi: $10.1136 / \mathrm{hrt} .40 .3 .256$

22. Domenichini F, Querzoli G, Cenedese A, Pedrizzetti G. Combined experimental and numerical analysis of the flow structure into the left ventricle. J. Biomech. 2007; 40(9): 1988-1994. doi: 10.1016/j.jbiomech.2006.09.024 